\title{
ON TWO CLASSES OF \\ WEIGHTED SOBOLEV-SLOBODETSKIĬ SPACES IN A DIHEDRAL ANGLE
}

\author{
J. ROSSMANN
}

Department of Mathematics, University of Rostock

Universitätsplatz 1, D-O-2500 Rostock, Germany

Introduction. In this paper some problems of the teory of weighted SobolevSlobodetskii spaces are dealt with. Weighted Sobolev spaces play an important role in the study of elliptic boundary value problems in non-smooth domains (see e.g. [2]-[4], [6]). Here two classes of weighted spaces are of special interest: the spaces $V_{p, \beta}^{l}(G)$ with the homogeneous norms

$$
\|u\|_{V_{p, \beta}^{l}(G)}=\left(\int_{G} \sum_{|\alpha| \leq l} r^{p(\beta-l+|\alpha|)}\left|D^{\alpha} u\right|^{p} d x\right)^{1 / p}
$$

( $r=r(x)$ denotes the distance of $x$ to the set of singularities on the boundary) and the spaces $W_{p, \beta}^{l}(G)$ with the inhomogeneous norms

$$
\|u\|_{W_{p, \beta}^{l}(G)}=\left(\int_{G} \sum_{|\alpha| \leq l} r^{p \beta}\left|D^{\alpha} u\right|^{p} d x\right)^{1 / p} .
$$

We restrict ourselves to the case that $G=\mathcal{D}$ is a dihedral angle. This is the model case for domains with smooth non-intersecting edges. For domains with conical points the weighted Sobolev spaces $V_{p, \beta}^{l}(G)$ and $W_{p, \beta}^{l}(G)$ were investigated e.g. in $[2],[5],[8]$.

The present paper consists of two sections. In Section 1 the main properties of the weighted Sobolev spaces $V_{p, \beta}^{l}(\mathcal{D}), W_{p, \beta}^{l}(\mathcal{D})(l$ an integer, $l \geq 0, \beta \in \mathbb{R}$, $1<p<\infty)$ and of the corresponding weighted Sobolev-Slobodetskiu spaces $V_{p, \beta}^{s}(\mathcal{D}), W_{p, \beta}^{s}(\mathcal{D})(s$ real, $s \geq 0, \beta \in \mathbb{R}, 1<p<\infty)$ will be investigated. It will be shown e.g. that the following imbeddings are valid:

$$
\begin{aligned}
& V_{p, \beta}^{s}(\mathcal{D}) \subset V_{p, \beta^{\prime}}^{s^{\prime}}(\mathcal{D}) \quad \text { if } s \geq s^{\prime}, s-\beta=s^{\prime}-\beta^{\prime}, \\
& W_{p, \beta}^{s}(\mathcal{D}) \subset W_{p, \beta^{\prime}}^{s^{\prime}}(\mathcal{D}) \quad \text { if } s \geq s^{\prime}, s-\beta=s^{\prime}-\beta^{\prime}, \beta^{\prime}>-2 / p \text {. }
\end{aligned}
$$


Furthermore, we give a connection between the spaces $V_{p, \beta}^{s}(\mathcal{D})$ and $W_{p, \beta}^{s}(\mathcal{D})$. It will be proved that every function $u \in W_{p, \beta}^{s}(\mathcal{D})$ can be written as the sum of a "quasi-polynomial" and a function from $V_{p, \beta}^{s}(\mathcal{D})$ (see Theorem 5). This is a generalization of Lemma 1.3 of [6] (see also [7]) where such a representation has been shown if $s$ is an integer and $\beta+2 / p$ is not an integer. Analogous results are obtained for the space $B_{p, \beta}^{s-1 / p}\left(\Gamma^{ \pm}\right)$and $B_{p, \beta}^{s-1 / p}\left(\Gamma^{ \pm}\right)$of the traces of functions from $V_{p, \beta}^{s}(\mathcal{D})$ and $W_{p, \beta}^{s}(\mathcal{D})$ on the sides $\Gamma^{+}$and $\Gamma^{-}$of $\mathcal{D}$, respectively.

Section 2 is concerned with two applications of the given results. In Section 2.1 we investigate conditions on $g_{k}^{ \pm} \in B_{p, \beta}^{s-m_{k}^{ \pm}-1 / p}\left(\Gamma^{ \pm}\right)$under which there exists $u \in$ $W_{p, \beta}^{s}(\mathcal{D})$ satisfying the boundary conditions

$$
B_{k}^{ \pm} u=g_{k}^{ \pm} \quad \text { on } \Gamma^{ \pm} \quad\left(k=1, \ldots, p^{ \pm}\right)
$$

where $\left\{B_{k}^{+}\right\}$and $\left\{B_{k}^{-}\right\}$are normal systems of homogeneous boundary operators of order $m_{k}^{ \pm}$with constant coefficients on $\Gamma^{+}$and $\Gamma^{-}$, respectively. In the spaces $V_{p, \beta}^{s}$ the existence of $u$ is always ensured (see [4]). We will show that $u \in W_{p, \beta}^{s}(\mathcal{D})$ satisfying (0.3) exists if and only if the boundary data $g_{k}^{ \pm}$satisfy some compatibility conditions on the edge $M$ (see Theorem 7 ).

In Section 2.2 the following regularity assertion for solutions of elliptic boundary value problems will be proved: If $u \in W_{p, \beta}^{2 m}(\mathcal{D})$ is a solution of an elliptic boundary value problem $L u=f$ in $\mathcal{D}, B_{k}^{ \pm} u=g_{k}^{ \pm}$on $\Gamma^{ \pm}(k=1, \ldots, m)$ where $f \in W_{p, \beta+s}^{s}(\mathcal{D}), g_{k}^{ \pm} \in B_{p, \beta+s}^{s+2 m-m_{k}^{ \pm}-1 / p}\left(\Gamma^{ \pm}\right)$then $u \in W_{p, \beta+s}^{s+2 m}(\mathcal{D})$.

The corresponding result for the spaces $V_{p, \beta}^{s}$ has been proved in [4].

\section{Weighted Sobolev-Slobodetskir spaces in a dihedral angle}

1.1. The spaces $V_{p, \beta}^{s}(\mathcal{D})$. Let $\mathcal{D}=K \times \mathbb{R}^{n-2}=\left\{x=(y, z) \in \mathbb{R}^{n}: y \in K\right.$, $\left.z=\left(z_{1}, \ldots, z_{n-2}\right) \in \mathbb{R}^{n-2}\right\}$ be a dihedral angle in $\mathbb{R}^{n}$ where $K$ is a plane wedge which has the following representation in polar coordinates $r, \omega$ :

$$
K=\left\{y=\left(y_{1}, y_{2}\right) \in \mathbb{R}^{2}: 0<r<\infty, \omega \in \Omega=\left(-\frac{1}{2} \omega_{0}, \frac{1}{2} \omega_{0}\right)\right\}
$$

$\left(0<\omega_{0} \leq 2 \pi\right)$. The boundary of $\mathcal{D}$ consists of the $(n-1)$-dimensional half-planes

$$
\Gamma^{ \pm}=\left\{x=(x, z) \in \mathbb{R}^{n}: 0<r<\infty, \omega= \pm \frac{1}{2} \omega_{0}, z \in \mathbb{R}^{n-2}\right\}
$$

and of the edge $M=\{(0,0)\} \times \mathbb{R}^{n-2}$. In the sequel we will denote the coordinates of a point $x=\left(x_{1}, \ldots, x_{n}\right)$ by $y_{1}, y_{2}, z_{1}, \ldots, z_{n-2}$, i.e. $y_{1}=x_{1}, y_{2}=x_{2}, z_{j}=$ $x_{j+2}(j=1, \ldots, n-2)$.

If $s$ is a non-negative integer and $p, \beta$ are real numbers, $1<p<\infty$, then $V_{p, \beta}^{s}(\mathcal{D})$ denotes the closure of $C_{0}^{\infty}(\overline{\mathcal{D}} \backslash M)=\left\{u \in C^{\infty}(\overline{\mathcal{D}}): \operatorname{supp} u \subset \overline{\mathcal{D}} \backslash M\right.$, $\operatorname{supp} u$ compact $\}$ with respect to the norm

$$
\|u\|_{V_{p, \beta}^{s}(\mathcal{D})}=\left(\int_{\mathcal{D}} \sum_{|\alpha| \leq s} r^{p(\beta-s+|\alpha|)}\left|D^{\alpha} u\right|^{p} d x\right)^{1 / p}
$$

$\left(D^{\alpha}=D_{x}^{\alpha}=D_{x_{1}}^{\alpha_{1}} \ldots D_{x_{n}}^{\alpha_{n}}, D_{x_{j}}=(1 / i) \partial_{x_{j}}=(1 / i) \frac{\partial}{\partial x_{j}}, r=|y|\right)$. 
For non-integer positive $s=l+\sigma(l$ an integer, $0<\sigma<1)$ the space $V_{p, \beta}^{s}(\mathcal{D})$ is defined as the closure of $C_{0}^{\infty}(\overline{\mathcal{D}} \backslash M)$ with respect to the norm

$$
\begin{aligned}
& \|u\|_{V_{p, \beta}^{s}(\mathcal{D})}=\left(\int_{\mathcal{D}} \sum_{|\alpha| \leq l}|y|^{p(\beta-s+|\alpha|)}\left|\left(D^{\alpha} u\right)(x)\right|^{p} d x\right. \\
& \left.\quad+\left.\int_{\mathcal{D}} \int_{\mathcal{D}} \sum_{|\alpha|=l}|| y\right|^{\beta}\left(D^{\alpha} u\right)(y, z)-\left.\left|y^{\prime}\right|^{\beta}\left(D^{\alpha} u\right)\left(y^{\prime}, z^{\prime}\right)\right|^{p} \frac{d x d x^{\prime}}{\left|x-x^{\prime}\right|^{n+p \sigma}}\right)^{1 / p} .
\end{aligned}
$$

It can easily be shown that the norm (1.2) is equivalent to the norm

$$
\begin{aligned}
& \|u\|=\left(\int_{\mathcal{D}} \sum_{|\alpha| \leq l}|y|^{p(\beta-s+|\alpha|)}\left|\left(D^{\alpha} u\right)(x)\right|^{p} d x\right. \\
& \left.\quad+\sum_{|\alpha|=l} \underset{\substack{\mathcal{D} \\
\left|x-x^{\prime}\right|<|y| / 2}}{\int}|y|^{p \beta}\left|\left(D^{\alpha} u\right)(y, z)-\left(D^{\alpha} u\right)\left(y^{\prime}, z^{\prime}\right)\right|^{p} \frac{d x d x^{\prime}}{\left|x-x^{\prime}\right|^{n+p \sigma}}\right)^{1 / p} .
\end{aligned}
$$

THEOREM 1. If $s^{\prime}>s$ and $\beta^{\prime}-s^{\prime}=\beta-s$ then $V_{p, \beta^{\prime}}^{s^{\prime}}(\mathcal{D})$ is continuously imbedded in $V_{p, \beta}^{s}(\mathcal{D})$.

Proof. If $l \leq s<s^{\prime}<l+1$ ( $l$ an integer) then the imbedding immediately follows from the inequality

$$
|y|^{\beta-\beta^{\prime}}=|y|^{s-s^{\prime}} \leq 2^{s-s^{\prime}}\left|x-x^{\prime}\right|^{s-s^{\prime}} \quad \text { for }\left|x-x^{\prime}\right|<\frac{1}{2}|y| .
$$

In the case $l<s<s^{\prime}=l+1$ we can apply the equation

$$
\begin{aligned}
\left(D^{\alpha} u\right)(x)-\left(D^{\alpha} u\right)\left(x^{\prime}\right) & =-\int_{0}^{1} \frac{d}{d t}\left(D^{\alpha} u\right)\left(x+t\left(x^{\prime}-x\right)\right) d t \\
& =\left(x-x^{\prime}\right) \int_{0}^{1}\left(\nabla D^{\alpha} u\right)\left(x+t\left(x^{\prime}-x\right)\right) d t
\end{aligned}
$$

to obtain

$$
\begin{aligned}
& \quad \int_{\substack{\mathcal{D} \\
\left|x-x^{\prime}\right|<|y| / 2}}|y|^{p \beta}\left|\left(D^{\alpha} u\right)(x)-\left(D^{\alpha} u\right)\left(x^{\prime}\right)\right|^{p} \frac{d x d x^{\prime}}{\left|x-x^{\prime}\right|^{n+p(s-l)}} \\
& \quad \leq \int_{0}^{1} \underset{\substack{\mathcal{D} \\
\left|x-x^{\prime}\right|<|y| / 2}}{\int}|y|^{p \beta}\left|x-x^{\prime}\right|^{-n+p(l+1-s)}\left|\left(\nabla D^{\alpha} u\right)\left(x+t\left(x-x^{\prime}\right)\right)\right|^{p} d x d x^{\prime} d t \\
& \leq \int_{\mathcal{D}}|y|^{p \beta^{\prime}}\left|\left(\nabla D^{\alpha} u\right)(x)\right|^{p} d x
\end{aligned}
$$

for $|\alpha|=l$, i.e. $\|u\|_{V_{p, \beta}^{s}(\mathcal{D})} \leq c\|u\|_{V_{p, \beta^{\prime}}^{s^{\prime}}(\mathcal{D})}$. 
In the sequel let $\zeta_{\nu}(\nu=\ldots,-1,0,+1, \ldots)$ be smooth functions on $\mathbb{R}_{+}$with support in the interval $\left[2^{\nu-1}, 2^{\nu+1}\right]$ such that

$$
\sum_{\nu=-\infty}^{\infty} \zeta_{\nu}(r)=1 \quad \text { and } \quad\left|D_{r}^{j} \zeta_{\nu}(r)\right|<c_{j} 2^{-\nu j}
$$

with constants $c_{j}$ independent of $r$ and $\nu$. If we set $r=|y|=\left(y_{1}^{2}+y_{2}^{2}\right)^{1 / 2}$ we can interpret $\zeta_{\nu}=\zeta_{\nu}(r)$ as functions on $\mathcal{D}$. Analogously to Lemma 1.1 in [4] the following assertion can be proved.

LEMMA 1. The norm $\|\cdot\|_{V_{p, \beta}^{s}(\mathcal{D})}$ is equivalent to the norm

$$
\|u\|=\left(\sum_{\nu=-\infty}^{\infty}\left\|\zeta_{\nu} u\right\|_{V_{p, \beta}^{s}(\mathcal{D})}^{p}\right)^{1 / p} .
$$

Let $\stackrel{\circ}{B}_{p, \beta}^{s-1 / p}\left(\Gamma^{ \pm}\right)(s>1 / p)$ be the space of the traces of functions from $V_{p, \beta}^{s}(\mathcal{D})$ on $\Gamma^{+}$and $\Gamma^{-}$, respectively, provided with the norm

$$
\|u\|_{\stackrel{\circ}{p, \beta}_{p, 1 / p}^{s-1}\left(\Gamma^{ \pm}\right)}=\inf \left\{\|v\|_{V_{p, \beta}^{s}(\mathcal{D})}: v \in V_{p, \beta}^{s}(\mathcal{D}),\left.v\right|_{\Gamma^{ \pm}}=u\right\} .
$$

Lemma 2. The norm (1.5) is equivalent to the norm

$$
\|u\|=\left(\sum_{\nu=-\infty}^{\infty}\left\|\zeta_{\nu} u\right\|_{B_{p, \beta}^{s-1 / p}\left(\Gamma^{ \pm}\right)}^{p}\right)^{1 / p} .
$$

Proof. Let $v \in V_{p, \beta}^{s}(\mathcal{D})$ be an extension of $u \in B_{p, \beta}^{s-1 / p}\left(\Gamma^{ \pm}\right)$such that $\|v\|_{V_{p, \beta}^{s}(\mathcal{D})} \leq 2\|u\|_{B_{p, \beta}^{s-1 / p}\left(\Gamma^{ \pm}\right)}$. Since $\zeta_{\nu} v$ is an extension of $\zeta_{\nu} u$ we get

$$
\begin{aligned}
\left(\sum_{\nu=-\infty}^{\infty}\left\|\zeta_{\nu} u\right\|_{B_{p, \beta}^{s-1 / p}\left(\Gamma^{ \pm}\right)}^{p}\right)^{1 / p} & \leq\left(\sum_{\nu=-\infty}^{\infty}\left\|\zeta_{\nu} v\right\|_{V_{p, \beta}^{s}(\mathcal{D})}^{p}\right)^{1 / p} \\
& \leq c\|v\|_{V_{p, \beta}^{s}(\mathcal{D})} \leq 2 c\|u\|_{B_{p, \beta}^{s-1 / p}\left(\Gamma^{ \pm}\right)}
\end{aligned}
$$

Furthermore, there exist extensions $v_{\nu}$ of $\zeta_{\nu} u$ such that $\left\|v_{\nu}\right\|_{V_{p, \beta}^{s}(\mathcal{D})} \leq$ $2\left\|\zeta_{\nu} u\right\|_{B_{p, \beta}^{s-1 / p}\left(\Gamma^{ \pm}\right)}(\nu=\ldots,-1,0,+1, \ldots)$. Since $w_{\nu}=\left(\zeta_{\nu-1}+\zeta_{\nu}+\zeta_{\nu+1}\right) v_{\nu}=\zeta_{\nu} u$ on $\Gamma^{ \pm}$and $w=\sum_{\nu=-\infty}^{\infty} w_{\nu}=u$ on $\Gamma^{ \pm}$we obtain

$$
\begin{aligned}
& \|u\|_{B_{p, \beta}^{s-1 / p}\left(\Gamma^{ \pm}\right)}^{p} \leq\|w\|_{V_{p, \beta}^{s}(\mathcal{D})}^{p} \leq c \sum_{\nu=-\infty}^{\infty}\left\|\zeta_{\nu} w\right\|_{V_{p, \beta}^{s}(\mathcal{D})}^{p}=c \sum_{\nu=-\infty}^{\infty}\left\|\zeta_{\nu} \sum_{k=-\infty}^{\infty} w_{k}\right\|_{V_{p, \beta}^{s}(\mathcal{D})}^{p} \\
& =c \sum_{\nu=-\infty}^{\infty}\left\|\zeta_{\nu} \sum_{k=\nu-2}^{\nu+2}\left(\zeta_{k-1}+\zeta_{k}+\zeta_{k+1}\right) v_{k}\right\|_{V_{p, \beta}^{s}(\mathcal{D})}^{p} \\
& \leq c^{\prime} \sum_{\nu=-\infty}^{\infty}\left\|v_{\nu}\right\|_{V_{p, \beta}^{s}(\mathcal{D})}^{p} \leq c^{\prime} 2^{p} \sum_{\nu=-\infty}^{\infty}\left\|\zeta_{\nu} u\right\|_{B_{p, \beta}^{s-1 / p}\left(\Gamma^{ \pm}\right)}^{p}
\end{aligned}
$$


In the half-planes $\Gamma^{+}, \Gamma^{-}$we introduce the Cartesian coordinates $\xi_{1}=r=$ $|y|=\operatorname{dist}(x, M), \xi_{2}=z_{1}, \ldots, \xi_{n-1}=z_{n-2}$. Using Lemmas 1 and 2 we can give the following norm equivalent to (1.5).

TheOREM 2. Let $p, \beta$ and $s(1<p<\infty, s>1 / p)$ be real numbers and $k$ an arbitrary integer such that $k>s-1 / p$. Then the norm (1.5) is equivalent to the norm

$$
\begin{aligned}
& \text { (1.6) } \quad\|u\|=\left(\int_{\mathbb{R}_{+} \times \mathbb{R}^{n-2}} \xi_{1}^{p(\beta-s)+1}|u(\xi)|^{p} d \xi\right. \\
& \left.+\int_{\substack{\mathbb{R}_{+} \times \mathbb{R}^{n-2} \\
\left|\xi-\xi^{\prime}\right|<\xi_{1} / 2}} \int_{\substack{\mathbb{R}_{+} \times \mathbb{R}^{n-2} \\
\mid}} \xi_{1}^{p \beta}\left|\sum_{j=0}^{k}(-1)^{j}\left(\begin{array}{c}
k \\
j
\end{array}\right) u\left(\frac{j \xi+(k-j) \xi^{\prime}}{k}\right)\right|^{p} \frac{d \xi d \xi^{\prime}}{\left|\xi-\xi^{\prime}\right|^{n-2+p s}}\right)^{1 / p} .
\end{aligned}
$$

Pro of. Let $u \in B_{p, \beta}^{s-1 / p}\left(\Gamma^{ \pm}\right)$. Define $u_{\nu}(\xi)=\zeta_{\nu}\left(2^{\nu} \xi\right) u\left(2^{\nu} \xi\right)(\nu=0, \pm 1, \pm 2, \ldots)$. Since supp $u_{\nu} \subset\left\{\xi \in \mathbb{R}_{+} \times \mathbb{R}^{n-2}: 1 / 2<\xi_{1}<2\right\}$ the norm of $u$ can be estimated by the usual Besov space norm (see [11]), i.e.

$$
\begin{aligned}
& c_{1}\left\|u_{\nu}\right\|_{B_{p, \beta}^{s-1 / p}\left(\Gamma^{ \pm}\right)}^{p} \leq \int_{\mathbb{R}_{+} \times \mathbb{R}^{n-2}}\left|u_{\nu}(\xi)\right|^{p} d \xi \\
& +\int_{\substack{\mathbb{R}_{+} \times \mathbb{R}^{n-2} \\
\left|\xi-\xi^{\prime}\right|<1 / 2}} \int_{\substack{\mathbb{R}_{+} \times \mathbb{R}^{n-2} \\
j}}\left|\sum_{j=0}^{k}(-1)^{j}\left(\begin{array}{c}
k \\
j
\end{array}\right) u_{\nu}\left(\frac{j \xi+(k-1) \xi^{\prime}}{k}\right)^{p} \frac{d \xi d \xi^{\prime}}{\left|\xi-\xi^{\prime}\right|^{n-2+p s}}\right| \\
& \leq c_{2}\left\|u_{\nu}\right\|_{B_{p, \beta}^{s-1 / p}\left(\Gamma^{ \pm}\right)}^{p}
\end{aligned}
$$

where the constants $c_{1}, c_{2}$ are independent of $u$ and $\nu$. It can be easily verified that $\left\|u_{\nu}\right\|_{B_{p, \beta}^{s s-1 / p}\left(\Gamma^{ \pm}\right)}=2^{\nu(s-\beta-n / p)}\left\|\zeta_{\nu} u\right\|_{B_{p, \beta}^{s-1 / p}\left(\Gamma^{ \pm}\right)}$. Hence,

$$
\begin{aligned}
& c_{1} 2^{\nu(p s-p \beta-n)}\left\|\zeta_{\nu} u\right\|_{\dot{B}_{p, \beta}^{o s-1 / p}\left(\Gamma^{ \pm}\right)}^{p} \leq 2^{-\nu(n-1)} \int_{\mathbb{R}_{+} \times \mathbb{R}^{n-2}}\left|\zeta_{\nu}(\xi) u(\xi)\right|^{p} d \xi \\
& +2^{-\nu(n-p s)} \int_{\substack{\mathbb{R}_{+} \times \mathbb{R}^{n-2} \\
\left|\xi-\xi^{\prime}\right|<\xi_{1} / 2}} \int_{\mathbb{R}_{+} \times \mathbb{R}^{n-2}} \mid \sum_{j=0}^{k}(-1)^{j}\left(\begin{array}{c}
k \\
j
\end{array}\right) \\
& \times\left.\zeta_{\nu}\left(\frac{j \xi+(k-j) \xi^{\prime}}{k}\right) u\left(\frac{j \xi+(k-j) \xi^{\prime}}{k}\right)\right|^{p} \frac{d \xi d \xi^{\prime}}{\left|\xi-\xi^{\prime}\right|^{n-2+p s}} \\
& \leq c_{2} 2^{\nu(p s-p \beta-n)}\left\|\zeta_{\nu} u\right\|_{B_{p, \beta}^{s-1 / p}\left(\Gamma^{ \pm}\right)}^{p} .
\end{aligned}
$$

Applying Lemma 2 we obtain the assertion of the theorem. 
Remark 1. By means of other equivalent norms in the usual Besov space (see [11], Section 4.4) analogously to Theorem 2 equivalence of other weighted norms to the norm (1.5) can be proved. In particular, if $s-1 / p$ is not an integer, $s-1 / p=l+\sigma(l$ an integer, $l \geq 0,0<\sigma<1)$ then (1.5) is equivalent to the norm

$$
\begin{aligned}
& \|u\|_{V_{p, \beta}^{s-1 / p}\left(\mathbb{R}_{+} \times \mathbb{R}^{n-2}\right)}=\left(\sum_{|\alpha| \leq l} \int_{\mathbb{R}_{+} \times \mathbb{R}^{n-2}} \xi_{1}^{p(\beta-s+|\alpha|)+1}\left|\left(D^{\alpha} u\right)(\xi)\right|^{p} d \xi\right. \\
& \left.\quad+\sum_{|\alpha|=l} \int_{\substack{\mathbb{R}_{+} \times \mathbb{R}^{n-2} \\
\left|\xi-\xi^{\prime}\right|<\xi_{1} / 2}} \int_{\mathbb{R}_{+} \times \mathbb{R}^{n-2}} \xi_{1}^{p \beta}\left|\left(D^{\alpha} u\right)(\xi)-\left(D^{\alpha} u\right)\left(\xi^{\prime}\right)\right|^{p} \frac{d \xi d \xi^{\prime}}{\left|\xi-\xi^{\prime}\right|^{n-1+p \sigma}}\right)^{1 / p}
\end{aligned}
$$

or to the norm

$$
\begin{aligned}
\|u\| & =\left(\int_{\mathbb{R}_{+} \times \mathbb{R}^{n-2}} \xi_{1}^{p(\beta-s)+1}|u(\xi)|^{p} d \xi\right. \\
& \left.+\sum_{|\alpha|=l} \int_{\substack{\mathbb{R}_{+} \times \mathbb{R}^{n-2} \\
\left|\xi-\xi^{\prime}\right|<\xi_{1} / 2}} \int_{\mathbb{R}_{+} \times \mathbb{R}^{n-2}} \xi_{1}^{p \beta}\left|\left(D^{\alpha} u\right)(\xi)-\left(D^{\alpha} u\right)\left(\xi^{\prime}\right)\right|^{p} \frac{d \xi d \xi^{\prime}}{\left|\xi-\xi^{\prime}\right|^{n-1+p \sigma}}\right)^{1 / p} .
\end{aligned}
$$

Analogously, the norm (1.2) is equivalent to the norm

$$
\begin{aligned}
\|u\|=\left(\int_{\mathcal{D}}|y|^{p(\beta-s)}|u(x)|^{p} d x\right. & \\
& \left.+\underset{\substack{\mathcal{D} \\
\mid}}{\quad} \int_{\mathcal{D}}|y|^{p \beta} \frac{\left|\left(D^{\alpha} u\right)(y, z)-\left(D^{\alpha} u\right)\left(y^{\prime}, z^{\prime}\right)\right|^{p}}{\left|x-x^{\prime}\right|^{n+p \sigma}} d x d x^{\prime}\right)^{1 / p} .
\end{aligned}
$$

1.2. The spaces $W_{p, \beta}^{s}(\mathcal{D})$. Let $p, \beta, s$ be real numbers, $1<p<\infty, \beta>-2 / p$, $s \geq 0$. If $s$ is an integer then we define the space $W_{p, \beta}^{s}(\mathcal{D})$ as the closure of $C_{0}^{\infty}(\overline{\mathcal{D}})$ with respect to the norm

$$
\|u\|_{W_{p, \beta}^{s}(\mathcal{D})}=\left(\sum_{|\alpha| \leq s} \int_{\mathcal{D}} r^{p \beta}\left|D^{\alpha} u\right|^{p} d x\right)^{1 / p} .
$$

If $s=l+\sigma(l$ an integer, $0<\sigma<1)$ then the space $W_{p, \beta}^{s}(\mathcal{D})$ will be defined as the closure of $C_{0}^{\infty}(\overline{\mathcal{D}})$ with respect to the norm

$$
\begin{aligned}
\|u\|_{W_{p, \beta}^{s}(\mathcal{D})}= & \left(\sum_{|\alpha| \leq l} \int_{\mathcal{D}} r^{p \beta}\left|D^{\alpha} u\right|^{p} d x\right. \\
& \left.+\sum_{|\alpha|=l} \underset{\substack{\mathcal{D} \\
\left|x-x^{\prime}\right|<|y| / 2}}{\int} \int^{p \beta} \frac{\left|\left(D^{\alpha} u\right)(x)-\left(D^{\alpha} u\right)\left(x^{\prime}\right)\right|^{p}}{\left|x-x^{\prime}\right|^{n+p \sigma}} d x d x^{\prime}\right)^{1 / p} .
\end{aligned}
$$


Furthermore, we define $W_{p, \beta}^{s}\left(\mathbb{R}_{+} \times \mathbb{R}^{n-2}\right)(s \geq 0,1<p<\infty, \beta>-1 / p)$ as the closure of $C_{0}^{\infty}\left(\overline{\mathbb{R}}_{+} \times \mathbb{R}^{n-2}\right)$ with respect to the norm

$$
\|u\|_{W_{p, \beta}^{s}\left(\mathbb{R}_{+} \times \mathbb{R}^{n-2}\right)}=\left(\int_{\mathbb{R}_{+} \times \mathbb{R}^{n-2}} r^{p \beta} \sum_{|\alpha| \leq s}\left|\left(D^{\alpha} u\right)(r, z)\right|^{p} d r d z\right)^{1 / p}
$$

if $s$ is an integer, and

$$
\begin{aligned}
& \|u\|_{W_{p, \beta}^{s}\left(\mathbb{R}_{+} \times \mathbb{R}^{n-2}\right)}=\left(\int_{\mathbb{R}_{+} \times \mathbb{R}^{n-2}} r^{p \beta} \sum_{|\alpha| \leq l}\left|\left(D^{\alpha} u\right)(r, z)\right|^{p} d r d z\right. \\
& \left.+\sum_{\substack {|\alpha|=l \\
\begin{subarray}{c}{\mathbb{R}_{+} \times \mathbb{R}^{n-2} \\
\left|\left(r-r^{\prime}, z-z^{\prime}\right)\right|<r / 2{ | \alpha | = l \\
\begin{subarray} { c } { \mathbb { R } _ { + } \times \mathbb { R } ^ { n - 2 } \\
| ( r - r ^ { \prime } , z - z ^ { \prime } ) | < r / 2 } }\end{subarray}} \int_{\substack{\mathbb{R}_{+} \times \mathbb{R}^{n-2} \\
\left(r^{\prime}\right.}} r^{p \beta} \frac{\left|\left(D^{\alpha} u\right)(r, z)-\left(D^{\alpha} u\right)\left(r^{\prime}, z^{\prime}\right)\right|^{p}}{\left|(r, z)-\left(r^{\prime}, z^{\prime}\right)\right|^{n-1+p \sigma}} d r d z d r^{\prime} d z^{\prime}\right)^{1 / p}
\end{aligned}
$$

if $s=l+\sigma(l$ an integer, $l \geq 0,0<\sigma<1)$.

Let $u=u\left(y_{1}, y_{2}, z\right)$ be an arbitrary function on $\mathcal{D}$. Then we denote by $\stackrel{i}{\text { the }}$ function

$$
\stackrel{\circ}{u}(r, z)=\frac{1}{\omega_{0}} \int_{-\omega_{0} / 2}^{\omega_{0} / 2} u(r \cos \omega, r \sin \omega, z) d \omega .
$$

LEMma 3. If $u \in W_{p, \beta}^{s}(\mathcal{D})$ then $\stackrel{i}{\in} W_{p, \beta+1 / p}^{s}\left(\mathbb{R}_{+} \times \mathbb{R}^{n-2}\right)$ and

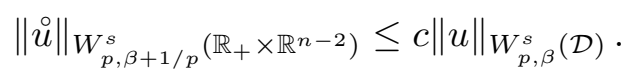

If $s$ is an integer then this assertion immediately follows from the definition of the spaces $W_{p, \beta}^{s}(\mathcal{D}), W_{p, \beta}^{s}\left(\mathbb{R}_{+} \times \mathbb{R}^{n-2}\right)$. For $s$ not an integer it is proved in [9].

We introduce the operator

$$
(\mathcal{K} g)(r, z)=\chi(r) \int_{\mathbb{R}^{n-2}} \int_{\mathbb{R}_{+}} g(t r, z+\tau r) K(t, \tau) d t d \tau
$$

where $\chi$ is a smooth cut-off function on $\overline{\mathbb{R}}_{+}$, equal to unity in $[0,1]$ and to zero in $(2, \infty)$ and $K(t, \tau)=\varphi(t) \psi\left(\tau_{1}\right) \ldots \psi\left(\tau_{n-2}\right)$ is a product of smooth functions $\varphi \in C_{0}^{\infty}\left(\mathbb{R}_{+}\right), \psi \in C_{0}^{\infty}(\mathbb{R})$ satisfying

$$
\begin{array}{ll}
\operatorname{supp} \varphi \subset(3 / 4,5 / 4), \quad & \int_{0}^{\infty} t^{j} \varphi(t) d t=\delta_{0, j}, \\
\operatorname{supp} \psi \subset(-1 / 4,1 / 4), \quad & \int_{-\infty}^{\infty} t^{j} \psi(t) d t=\delta_{0, j}
\end{array}
$$

$(j=0,1, \ldots, k)$. Here $\delta_{0, j}$ denotes the Kronecker symbol. 
LEMmA 4. If $g \in W_{p, \beta}^{s}\left(\mathbb{R}_{+} \times \mathbb{R}^{n-2}\right)$ then $\mathcal{K} g \in \bigcap_{\nu=1}^{\infty} W_{p, \beta+\langle s\rangle-s+\nu}^{\langle s\rangle+\nu}\left(\mathbb{R}_{+} \times \mathbb{R}^{n-2}\right)$. Furthermore,

$$
\int_{\mathbb{R}^{n-2}} \int_{\mathbb{R}_{+}} r^{p(\beta-s+j+|\gamma|)}\left|D_{r}^{j} D_{z}^{\gamma}(\mathcal{K} g)(r, z)\right|^{p} d r d z \leq c\|g\|_{W_{p, \beta}^{s}}^{p}\left(\mathbb{R}_{+} \times \mathbb{R}^{n-2}\right)
$$

for $j \geq 1$ or $|\gamma| \geq\langle s\rangle+1$, and

$$
\iint_{\mathbb{R}^{n-2}} r_{\mathbb{R}_{+}}^{p(\beta-s+\langle s\rangle)+1}\left|D_{z}^{\gamma}(\mathcal{K} g-g)\right|^{p} d r d z \leq c\|g\|_{W_{p, \beta}^{s}\left(\mathbb{R}_{+} \times \mathbb{R}^{n-2}\right)}^{p}
$$

for $|\gamma|=\langle s\rangle$. Here $\langle s\rangle$ denotes the largest integer less than $s$.

Pr o of. For simplicity we restrict ourselves to the case $n=3$. For $n>3$ the lemma can be proved analogously.

If $j+|\gamma|=\langle s\rangle$ and $r<1$ then $D_{r}^{j} D_{z}^{\gamma}(\mathcal{K} g)(r, z)$ is a linear combination of terms of the form

$$
\begin{aligned}
& T=\int_{\mathbb{R}} \int_{\mathbb{R}_{+}}\left(D^{\alpha} g\right)(t r, z+\tau r) t^{\mu} \varphi(t) \tau^{\nu} \psi(\tau) d t d \tau \\
& =r^{-2} \int_{\mathbb{R}} \int_{\mathbb{R}_{+}}\left(D^{\alpha} g\right)(t, \tau)\left(\frac{t}{r}\right)^{\mu} \varphi\left(\frac{t}{r}\right)\left(\frac{\tau-z}{r}\right)^{\nu} \psi\left(\frac{\tau-z}{r}\right) d t d \tau
\end{aligned}
$$

where $|\alpha|=j+|\gamma|, \mu+\nu=j$.

Let $j+|\gamma| \geq\langle s\rangle+1, r<1$. Since

$$
\int_{\mathbb{R}} \int_{\mathbb{R}_{+}} r^{-2}\left(\frac{t}{r}\right)^{\mu} \varphi\left(\frac{t}{r}\right)\left(\frac{\tau-z}{r}\right)^{\nu} \psi\left(\frac{\tau-z}{r}\right) d t d \tau=\int_{\mathbb{R}} \int_{\mathbb{R}_{+}} t^{\mu} \varphi(t) \tau^{\nu} \psi(\tau) d t d \tau
$$

is a constant, the function $D_{r}^{j} D_{z}^{\gamma} \mathcal{K} g$ can be written as a finite sum of expressions of the form

$$
\begin{aligned}
& T^{\prime}=c r^{-2+\langle s\rangle-j-|\gamma|} \int_{\mathbb{R}} \int_{\mathbb{R}_{+}}\left(\left(D^{\alpha} g\right)(t, \tau)-\left(D^{\alpha} g\right)(r, z)\right) \\
& \times\left(\frac{t}{r}\right)^{\mu_{1}} \varphi^{\left(\mu_{2}\right)}\left(\frac{t}{r}\right)\left(\frac{\tau-z}{r}\right)^{\nu_{1}} \psi^{\left(\nu_{2}\right)}\left(\frac{\tau-z}{r}\right) d t d \tau \\
&\left.=c r^{\langle s\rangle-j-|\gamma|} \int_{\mathbb{R}_{\mathbb{R}_{+}} \int_{\mathbb{R}^{\prime}}\left(\left(D^{\alpha} g\right)(t r, z+\tau r)-\right.}\left(D^{\alpha} g\right)(r, z)\right) \\
& \times t^{\mu_{1}} \varphi^{\left(\mu_{2}\right)}(t) \tau^{\nu_{1}} \psi^{\left(\nu_{2}\right)}(\tau) d t d \tau
\end{aligned}
$$

where $|\alpha|=\langle s\rangle$. Consequently, for $j+|\gamma| \geq\langle s\rangle+1$ we obtain

$$
\int_{\mathbb{R}} \int_{0}^{1} r^{p(\beta-s+j+|\gamma|)}\left|D_{r}^{j} D_{z}^{\gamma}(\mathcal{K} g)(r, z)\right|^{p} d r d z
$$




$$
\begin{aligned}
& \leq c \sum_{|\alpha|=s} \int_{\mathbb{R}} \int_{\mathbb{R}_{+}} r^{p(\beta-\sigma)} \int_{-1 / 4}^{1 / 4} \int_{3 / 4}^{5 / 4}\left|\left(D^{\alpha} g\right)(t r, z+\tau r)-\left(D^{\alpha} g\right)(r, z)\right|^{p} d t d \tau d r d z \\
& =c \sum_{|\alpha|=s} \int_{\mathbb{R}_{\mathbb{R}_{+}}} r^{p(\beta-\sigma)-2}\left(\int_{z-r / 4}^{z+r / 4} \int_{3 r / 4}^{5 r / 4}\left|\left(D^{\alpha} g\right)\left(r^{\prime}, z^{\prime}\right)-\left(D^{\alpha} g\right)(r, z)\right|^{p} d r^{\prime} d z^{\prime}\right) d r d z \\
& \leq c \sum_{|\alpha|=s} \int_{\mathbb{R}_{\mathbb{R}_{+}}} r^{p \beta}\left(\int_{\mathbb{R}_{\mathbb{R}_{+}}} \frac{\left|\left(D^{\alpha} g\right)\left(r^{\prime}, z^{\prime}\right)-\left(D^{\alpha} g\right)(r, z)\right|^{p}}{\left(\left|r-r^{\prime}\right|^{2}+\left|z-z^{\prime}\right|^{2}\right)^{1+p \sigma / 2}} d r^{\prime} d z^{\prime}\right) d r d z \\
& \leq c\|g\|_{W_{p, \beta}^{s}\left(\mathbb{R}_{+} \times \mathbb{R}^{n-2}\right)}^{p} \cdot
\end{aligned}
$$

Analogously, the inequality (1.14) can be proved. Furthermore, it can be shown that $r^{\beta} D_{z}^{\alpha} \mathcal{K} g \in L_{p}\left(\mathbb{R}_{+} \times \mathbb{R}^{n-2}\right)$. Together with (1.13) and (1.14) this implies $\mathcal{K} g \in \bigcap_{\nu=1}^{\infty} W_{p, \beta+\langle s\rangle-s+\nu}^{\langle s\rangle+\nu}\left(\mathbb{R}_{+} \times \mathbb{R}^{n-2}\right)$.

Remark 2. If we interpret $\mathcal{K} g$ as a function on $\mathcal{D}$ (i.e. we define $v(y, z)=$ $(\mathcal{K} g)(|y|, z)=(\mathcal{K} g)(r, z))$ then

$$
\mathcal{K} g \in \bigcap_{\nu=1}^{\infty} W_{p, \beta+\langle s\rangle-s+\nu-1 / p}^{\langle s\rangle+\nu}(\mathcal{D}) \quad \text { for } g \in W_{p, \beta}^{s}\left(\mathbb{R}_{+} \times \mathbb{R}^{n-2}\right)
$$

The following lemma is a consequence of the Hardy inequality

$$
\int_{0}^{\infty} r^{\beta-p}|f(r)|^{p} d r \leq\left(\frac{p}{|\beta-p+1|}\right)^{p} \int_{0}^{\infty} r^{\beta}\left|f^{\prime}(r)\right|^{p} d r
$$

which is satisfied if $f(0)=0, \beta<p-1$ or if $f(\infty)=0, \beta>p-1$.

LEMma 5. Let $u \in W_{p, \beta}^{0}(\mathcal{D})(\beta>-2 / p)$ be such that $\nabla u \in W_{p, \beta^{\prime}}^{0}(\mathcal{D})$ where $\beta^{\prime}>1-2 / p$. Then $u \in V_{p, \beta^{\prime}}^{1}(\mathcal{D})$.

Now we can prove an imbedding analogous to Theorem 1 for the spaces $W_{p, \beta}^{s}(\mathcal{D})$.

THEOREM 3. Let $s^{\prime} \geq s, \beta^{\prime}-s^{\prime}=\beta-s$ and $\beta>-2 / p$. Then $W_{p, \beta^{\prime}}^{s^{\prime}}(\mathcal{D})$ is continuously imbedded in $W_{p, \beta}^{s}(\mathcal{D})$.

Proof. Without loss of generality we can assume that $l \leq s<s^{\prime} \leq l+1$ where $l$ is a non-negative integer. We consider the following cases: (a) $s=l, s^{\prime}=l+\sigma$ $(0<\sigma<1)$, (b) $s=l+\sigma, s^{\prime}=l+\sigma^{\prime}\left(0<\sigma<\sigma^{\prime}<1\right),(\mathrm{c}) s=l+\sigma, s^{\prime}=l+1$ $(0<\sigma<1)$.

(a) Let $u \in W_{p, \beta^{\prime}}^{s^{\prime}}(\mathcal{D})$ and $v_{\alpha}=D^{\alpha} u(|\alpha|=l)$. By Lemma 5 and (1.14) we have $\mathcal{K} \dot{\vartheta}_{\alpha} \in W_{p, \beta+1-\sigma}^{1}(\mathcal{D})=W_{p, \beta+1}^{1}(\mathcal{D}) \subset W_{p, \beta}^{0}(\mathcal{D})$ and $v_{\alpha}-\mathcal{K} \dot{v}_{\alpha} \in W_{p, \beta}^{0}(\mathcal{D})$. Hence, $v_{\alpha}=D^{\alpha} u \in W_{p, \beta}^{0}(\mathcal{D})$ and by Lemma 5 we obtain $D^{\alpha} u \in W_{p, \beta}^{0}(\mathcal{D})$ for $|\alpha| \leq l$. 
(b) Analogously to (a) we obtain $\mathcal{K} \dot{v}_{\alpha} \in W_{p, \beta^{\prime}+1-\sigma^{\prime}}^{1}(\mathcal{D})=W_{p, \beta+1-\sigma}^{1}(\mathcal{D})$ for $|\alpha|=l$. Since $\mathcal{K} \stackrel{\circ}{\alpha}_{\alpha}=0$ for $r=|y|>2$ this implies $\mathcal{K} \dot{\vartheta}_{\alpha} \in W_{p, \beta+1}^{1}(\mathcal{D}) \subset W_{p, \beta}^{0}(\mathcal{D})$ for $|\alpha|=l$. Furthermore, by $(1.14), v_{\alpha}-\mathcal{K} \dot{v}_{\alpha} \in W_{p, \beta-\sigma}^{0}(\mathcal{D}) \cap W_{p, \beta^{\prime}}^{0}(\mathcal{D}) \subset W_{p, \beta}^{0}(\mathcal{D})$ and analogously to (a) it follows that $D^{\alpha} u \in W_{p, \beta}^{0}(\mathcal{D})$ for $|\alpha| \leq l$. Using the fact that $\beta-\beta^{\prime}=\sigma-\sigma^{\prime}$ we get

$$
\begin{aligned}
& \iint_{\substack{\mathcal{D} \\
\left|x-x^{\prime}\right|<|y| / 2}}|y|^{p \beta}\left|\left(D^{\alpha} u\right)(x)-\left(D^{\alpha} u\right)\left(x^{\prime}\right)\right|^{p} \frac{d x d x^{\prime}}{\left|x-x^{\prime}\right|^{n+p \sigma}} \\
& \quad \leq c \quad \int_{\substack{\mathcal{D} \\
\left|x-x^{\prime}\right|<|y| / 2}}|y|^{p \beta^{\prime}}\left|\left(D^{\alpha} u\right)(x)-\left(D^{\alpha} u\right)\left(x^{\prime}\right)\right|^{p} \frac{d x d x^{\prime}}{\left|x-x^{\prime}\right|^{n+p \sigma^{\prime}}}
\end{aligned}
$$

for $|\alpha|=l$. Hence, $u \in W_{p, \beta}^{s}(\mathcal{D})$.

(c) By Lemma 5 the space $W_{p, \beta^{\prime}}^{l+1}(\mathcal{D})$ is imbedded in $W_{p, \beta}^{l}(\mathcal{D})$. Furthermore, the equation

$$
\left(D^{\alpha} u\right)(x)-\left(D^{\alpha} u\right)\left(x^{\prime}\right)=\left(x-x^{\prime}\right) \int_{0}^{1}\left(\nabla D^{\alpha} u\right)\left(x+t\left(x^{\prime}-x\right)\right) d t
$$

yields

$$
\begin{aligned}
\quad \int_{\substack{\mathcal{D} \\
\left|x-x^{\prime}\right|<|y| / 2}}|y|^{p \beta}\left|\left(D^{\alpha} u\right)(x)-\left(D^{\alpha} u\right)\left(x^{\prime}\right)\right|^{p} \frac{d x d x^{\prime}}{\left|x-x^{\prime}\right|^{n+p \sigma}} \\
\leq c \int_{\mathcal{D}}|y|^{p \beta^{\prime}} \sum_{\left|\alpha^{\prime}\right|=l+1}\left|\left(D^{\alpha^{\prime}} u\right)(x)\right|^{p} d x
\end{aligned}
$$

for $|\alpha|=l$. This implies $W_{p, \beta^{\prime}}^{l+1}(\mathcal{D}) \subset W_{p, \beta}^{s}(\mathcal{D})$.

Corollary 1. If $\beta>s-2 / p$ then

$$
W_{p, \beta}^{s}(\mathcal{D}) \subset W_{p, \beta-s+\langle s\rangle}^{\langle s\rangle}(\mathcal{D}) \subset W_{p, \beta-s+\langle s\rangle-1}^{\langle s\rangle-1}(\mathcal{D}) \subset \ldots \subset W_{p, \beta-s}^{0}(\mathcal{D}),
$$

i.e. $W_{p, \beta}^{s}(\mathcal{D}) \subset V_{p, \beta}^{s}(\mathcal{D})$.

Remark 3. Analogously to Theorem 3 it can be proved that $W_{p, \beta^{\prime}}^{s^{\prime}}\left(\mathbb{R}_{+} \times\right.$ $\left.\mathbb{R}^{n-2}\right)$ is continuously imbedded in $W_{p, \beta}^{s}(\mathcal{D})$ if $s^{\prime} \geq s, \beta^{\prime}-s^{\prime}=\beta-s, \beta>-1 / p$.

1.3. Traces of functions from $W_{p, \beta}^{s}(\mathcal{D})$ on $M$. In [6] (Lemma 1.1) it has been proved that the trace of a function $u \in W_{p, \beta}^{l}(\mathcal{D})$ belongs to the Besov space $B_{p}^{l-\beta-2 / p}(M)$ for $l$ an integer, $l>\beta+2 / p>0$. Here the norm in $B_{p}^{\varkappa}(M)$ is defined by

$$
\|u\|_{B_{p}^{\varkappa(M)}}=\left(\|u\|_{L_{p}(M)}^{p}+\int_{M} \int_{M}\left|\Delta_{\zeta}^{k} u(z)\right|^{p} \frac{d z d \zeta}{|\zeta|^{n-2+p \varkappa}}\right)^{1 / p}
$$

where $\Delta_{\zeta}^{k} u(z)=\sum_{\nu=0}^{k}(-1)^{\nu}\left(\begin{array}{l}k \\ \nu\end{array}\right) u(z+\nu \zeta)$. 
THEOREM 4. Let $u \in W_{p, \beta}^{s}(\mathcal{D}), s>\beta+2 / p>0$. Then the trace of $u$ on $M$ exists and belongs to the Besov space $B_{p}^{s-\beta-2 / p}(M)$.

Proof. Let $\left\{u_{n}\right\}$ be a sequence of functions from $C_{0}^{\infty}(\overline{\mathcal{D}})$ which converges to $u$ in $W_{p, \beta}^{s}(\mathcal{D})$. Furthermore, let $\left\{f_{n}\right\}$ be the sequence of the traces of $u_{n}$ on $M$, i.e. $f_{n}(z)=u_{n}(0,0, z)=\stackrel{\circ}{u}_{n}(0, z)$ (the function $\stackrel{\circ}{n}_{n}$ is defined by (1.11)). Since the functions $\stackrel{\circ}{n}_{n}$ can be interpreted as functions from $W_{p,-1 / p+1 / 2}^{1}\left(\mathbb{R}_{+} \times \mathbb{R}^{n-2}\right)$ Lemma 4 yields

$$
\int_{\mathbb{R}_{+} \times \mathbb{R}^{n-2}} r^{-1-p / 2}\left|\stackrel{\circ}{n}_{n}(r, z)-\left(\mathcal{K} \dot{\circ}_{n}\right)(r, z)\right|^{p} d r d z<\infty .
$$

Consequently, $\stackrel{\leftrightarrow}{u}_{n}(0, z)-\left(\mathcal{K} \stackrel{\circ}{u}_{n}\right)(0, z)$, i.e. $f_{n}$ is the trace of $\mathcal{K} \stackrel{\circ}{n}_{n}$ on $M$. We denote the trace of $\mathcal{K} u$ on $M$ by $f$. Then by Lemma 1.1 of [6] and Lemmas 3 and 4 we get

$$
\begin{aligned}
\left\|f-f_{n}\right\|_{B_{p}^{s-\beta-2 / p}(M)} & \leq c\left\|\mathcal{K} \stackrel{\circ}{u}-\mathcal{K} \stackrel{\circ}{n}_{n}\right\|_{W_{p, \beta-s+\langle s\rangle+1}^{\langle s\rangle+1}}(\mathcal{D}) \\
& \leq c^{\prime}\left\|u-u_{n}\right\|_{W_{p, \beta}^{s}(\mathcal{D})}
\end{aligned}
$$

Hence, $\left\{f_{n}\right\}$ converges to $f$ in $B_{p}^{s-\beta-2 / p}(M)$.

Conversely, it can be proved that every $f \in B_{p}^{s-\beta-2 / p}(M)(s>\beta+2 / p>0)$ can be extended to a function $v \in W_{p, \beta}^{s}(\mathcal{D})$. We define the extension operator $\mathfrak{K}$ as follows:

$$
(\mathfrak{K} f)(r, z)=\chi(r) \int_{\mathbb{R}^{n-2}} g(z+\tau r) \psi\left(\tau_{1}\right) \ldots \psi\left(\tau_{n-2}\right) d \tau
$$

where $\psi \in C_{0}^{\infty}(\mathbb{R})$ satisfies (1.12). By Lemma 1.2 of [6] the operator $\mathfrak{K}$ defines a continuous map from $B_{p}^{\varkappa}(M)$ into $W_{p, s-\varkappa-2 / p}^{s}(\mathcal{D})$ for $\varkappa>0, \varkappa$ not an integer, $s$ an integer, $s+\langle-\varkappa-2 / p\rangle>-2$. From Theorem 3 it follows that this is also true for real $s, s+\langle-\varkappa-2 / p\rangle>-2$.

1.4. Connection between $V_{p, \beta}^{s}(\mathcal{D})$ and $W_{p, \beta}^{s}(\mathcal{D})$. In [6], [7] it has been proved that every $u \in W_{p, \beta}^{s}(\mathcal{D})$ is the sum of a "quasi-polynomial" and a function from $V_{p, \beta}^{s}(\mathcal{D})$ if $s$ is a non-negative integer and $\beta+2 / p$ is not an integer. We will give a similar connection between $V_{p, \beta}^{s}(\mathcal{D})$ and $W_{p, \beta}^{s}(\mathcal{D})$ without any restrictions on $s$ and $\beta$.

Let $u \in W_{p, \beta}^{s}(\mathcal{D})(\beta>-2 / p)$. We denote the derivatives $\partial_{y_{1}}^{i} \partial_{y_{2}}^{j} u(i+j \leq\langle s\rangle)$ by $u_{i j}$. By using the properties of the operator $\mathcal{K}$ the following lemma can be easily proved (see [9]).

LEMMA 6. If $u \in W_{p, \beta}^{s}(\mathcal{D})$ then the following inequality holds for $i+j+|\alpha| \leq\langle s\rangle$ :

$$
\begin{array}{r}
\int_{\mathcal{D}} r^{p(\beta-s+i+j+|\alpha|)}\left|\partial_{y_{1}}^{i} \partial_{y_{2}}^{j} \partial_{z}^{\alpha} u-\sum_{\mu+\nu=0}^{\langle s\rangle-i-j-|\alpha|}\left(\partial_{z}^{\alpha} \mathcal{K} \stackrel{\circ}{i+\mu, j+\nu}\right)_{\mu} \frac{y_{1}^{\mu} y_{2}^{\nu}}{\mu ! \nu !}\right|^{p} d x \\
\leq c\|u\|_{W_{p, \beta}^{s}(\mathcal{D})}^{p} .
\end{array}
$$


Lemma 6 implies the following corollary.

Corollary 2. If $u \in W_{p, \beta}^{s}(\mathcal{D})$ then

$$
u-\sum_{i+j=0}^{\langle s\rangle}\left(\mathcal{K} \stackrel{\circ}{i j}_{i j}\right)(r, z) \frac{y_{1}^{i} y_{2}^{j}}{i ! j !} \in V_{p, \beta}^{s}(\mathcal{D}) .
$$

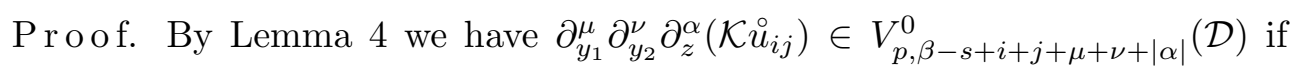
$\mu+\nu \geq 1$ or $|\alpha| \geq s-i-j$. Therefore, from Lemma 6 it follows that

$$
\int_{\mathcal{D}} r^{p(\beta-s+\mu+\nu+|\alpha|)}\left|\partial_{y_{1}}^{\mu} \partial_{y_{2}}^{\nu} \partial_{z}^{\alpha}\left(u-\sum_{i+j=0}^{\langle s\rangle}\left(\mathcal{K} \stackrel{\circ}{i j}_{i j}\right) \frac{y_{1}^{i} y_{2}^{j}}{i ! j !}\right)\right|^{p} d x<\infty
$$

for $\mu+\nu+|\alpha| \leq\langle s\rangle$, which yields the desired conclusion.

By Corollary 2 for every $u \in W_{p, \beta}^{s}(\mathcal{D})$ there exist $v_{i j} \in W_{p, \beta+1 / p}^{s}\left(\mathbb{R}_{+} \times \mathbb{R}^{n-2}\right)$ such that

$$
u-\sum_{i+j=0}^{\langle s\rangle}\left(\mathcal{K} v_{i j}\right)(r, z) \frac{y_{1}^{i} y_{2}^{j}}{i ! j !} \in V_{p, \beta}^{s}(\mathcal{D}) .
$$

Now we investigate the question whether the $v_{i j}$ are (in some sense) uniquely determined by $u$. Similarly to [8] we introduce the following equivalence relation in $W_{p, \beta+1 / p}^{s}\left(\mathbb{R}_{+} \times \mathbb{R}^{n-2}\right)(s>0, \beta>-2 / p)$ :

$$
f^{\beta-s, p} g \Leftrightarrow \int_{\mathbb{R}_{+} \times \mathbb{R}^{n-2}} r^{p(\beta-s)+1}|(\mathcal{K} f)(r, z)-(\mathcal{K} g)(r, z)|^{p} d r d z<\infty .
$$

Another characterization is the following:

$$
f^{\beta-s, p} g \Leftrightarrow \mathcal{K}(f-g) \in \bigcap_{\nu=0}^{\infty} V_{p, \beta-s+\nu+1 / p}^{\nu}\left(\mathbb{R}_{+} \times \mathbb{R}^{n-2}\right) .
$$

Proof. If $f, g \in W_{p, \beta+1 / p}^{s}\left(\mathbb{R}_{+} \times \mathbb{R}^{n-2}\right)$ and $f \stackrel{\beta-s, p}{\sim} g$ then by Lemma 4

$$
\mathcal{K}(f-g) \in \bigcap_{\nu=\langle s\rangle+1}^{\infty} W_{p, \beta-s+\nu+1 / p}^{\nu}\left(\mathbb{R}_{+} \times \mathbb{R}^{n-2}\right) \cap V_{p, \beta-s+1 / p}^{0}\left(\mathbb{R}_{+} \times \mathbb{R}^{n-2}\right),
$$

and (1.17) follows from $W_{p, \beta}^{l}\left(\mathbb{R}_{+} \times \mathbb{R}^{n-2}\right) \cap V_{p, \beta-l}^{0}\left(\mathbb{R}_{+} \times \mathbb{R}^{n-2}\right) \subset V_{p, \beta}^{l}\left(\mathbb{R}_{+} \times \mathbb{R}^{n-2}\right)$ (cf. Remark 1).

Remark 4. Let $f, g \in W_{p, \beta+1 / p}^{s}\left(\mathbb{R}_{+} \times \mathbb{R}^{n-2}\right)$.

(a) If $\beta-s<-2 / p$ then $\left.f \stackrel{\beta-s, p}{\sim} g \Leftrightarrow f\right|_{M}=\left.g\right|_{M}$.

(b) If $\beta-s=-2 / p$ then

$$
f^{\beta-s, p} g \Leftrightarrow \int_{\mathbb{R}_{+} \times \mathbb{R}^{n-2}} r^{p(\beta-s)+1}|f(r, z)-g(r, z)|^{p} d r d z<\infty .
$$


(c) If $\beta-s>-2 / p$ then $f \stackrel{\beta-s, p}{\sim} g \stackrel{\beta-s, p}{\sim} 0$.

Proof. (a) If $\beta-s<-2 / p$ then the trace of $f-g$ on $M$ exists and coincides with that of $\mathcal{K}(f-g)$. The Hardy inequality and Lemma 4 imply

$$
\begin{aligned}
& \left.\int_{\mathbb{R}_{+} \times \mathbb{R}^{n-2}} r^{p(\beta-s)+1}|\mathcal{K}(f-g)-(f-g)|_{M}\right|^{p} d r d z \\
& \leq c \quad \int_{\mathbb{R}_{+} \times \mathbb{R}^{n-2}} r^{p(\beta-s+1)+1}\left|\frac{\partial}{\partial r} \mathcal{K}(f-g)\right|^{p} d r d z \leq c\|f-g\|_{W_{p, \beta+1 / p}^{s}}^{p}\left(\mathbb{R}_{+} \times \mathbb{R}^{n-2}\right) .
\end{aligned}
$$

Hence, from (1.16) it follows that

$$
\left.\int_{\mathbb{R}_{+} \times \mathbb{R}^{n-2}} r^{p(\beta-s)+1}|(f-g)|_{M}\right|^{p} d r d z<\infty,
$$

i.e. $\left.f\right|_{M}=\left.g\right|_{M}$. Conversely, if $\left.f\right|_{M}=\left.g\right|_{M}$ then (1.16) follows from (1.18).

(b) If $\beta-s=-2 / p$ then $W_{p, \beta+1 / p}^{s}\left(\mathbb{R}_{+} \times \mathbb{R}^{n-2}\right) \subset W_{p, \beta-\langle s\rangle+1 / p}^{s-\langle s\rangle}\left(\mathbb{R}_{+} \times \mathbb{R}^{n-2}\right)$ (see Remark 3) and Lemma 4 yields

$$
\begin{aligned}
& \int_{\mathbb{R}_{+} \times \mathbb{R}^{n-2}} r^{p(\beta-s)+1}|\mathcal{K} f-f|^{p} d r d z \leq\|\| f \|_{W_{p, \beta-\langle s\rangle+1 / p}^{s-\langle s\rangle}}^{p}\left(\mathbb{R}_{+} \times \mathbb{R}^{n-2}\right) \\
& \leq c\|f\|_{W_{p, \beta+1 / p}^{s}}^{p}\left(\mathbb{R}_{+} \times \mathbb{R}^{n-2}\right)
\end{aligned}
$$

This proves the assertion.

(c) If $\beta-s>-2 / p$ then $W_{p, \beta+1 / p}^{s}\left(\mathbb{R}_{+} \times \mathbb{R}^{n-2}\right)$ is imbedded in $V_{p, \beta+1 / p}^{s}\left(\mathbb{R}_{+} \times\right.$ $\mathbb{R}^{n-2}$ ) (cf. Corollary 1, Remark 3). Consequently, (1.16) is satisfied for all $f, g \in$ $W_{p, \beta+1 / p}^{s}\left(\mathbb{R}_{+} \times \mathbb{R}^{n-2}\right)$.

LEMMA 7. Let $f \in W_{p, \beta+1 / p}^{s}\left(\mathbb{R}_{+} \times \mathbb{R}^{n-2}\right), \beta-s \leq-1-2 / p$. Then $f \stackrel{\beta-s, p}{\sim} 0$ iff $\partial f / \partial z_{i} \stackrel{\beta-s+1, p}{\sim} 0$ for one $i \in\{1, \ldots, n-2\}$.

Proof. 1) If $f \stackrel{\beta-s, p}{\sim} 0$ then $\mathcal{K} f \in V_{p, \beta+1 / p}^{s}\left(\mathbb{R}_{+} \times \mathbb{R}^{n-2}\right)$ and

$$
\mathcal{K} \frac{\partial f}{\partial z_{i}}=\frac{\partial}{\partial z_{i}} \mathcal{K} f \in V_{p, \beta+1 / p}^{s-1}\left(\mathbb{R}_{+} \times \mathbb{R}^{n-2}\right),
$$

i.e. $\partial f / \partial z_{i} \stackrel{\beta-s+1, p}{\sim} 0$.

2) Let $\partial f / \partial z_{1} \stackrel{\beta-s+1, p}{\sim} 0$. If $\beta-s<-1-2 / p$ this means $\frac{\partial}{\partial z_{1}}\left(\left.f\right|_{M}\right)=\left.\frac{\partial f}{\partial z_{1}}\right|_{M}=0$ where $\left.f\right|_{M} \in B_{p}^{s-\beta-2 / p}(M)$. Hence, $\left.f\right|_{M}=0$, i.e. $f \stackrel{\beta-s, p}{\sim} 0$. If $\beta-s=-1-2 / p$ we first assume that $f(r, z)=0$ for $r>1$ and $\left|z_{1}\right|>1$. Using the Hardy inequality we get

$\int_{\mathbb{R}_{+} \times \mathbb{R}^{n-2}} r^{-1}|f(0, z)|^{p} d r d z \leq c \int_{\mathbb{R}_{+} \times \mathbb{R}^{n-2}}\left(r^{-1}|f(r, z)|^{p}+r^{-2}|f(r, z)-f(0, z)|^{p}\right) d r d z$ 


$$
\begin{aligned}
& \leq c \int_{\mathbb{R}_{+} \times \mathbb{R}^{n-2}}\left(r^{-1}\left|\int_{-1}^{z_{1}} \frac{\partial}{\partial t} f\left(r, t, z_{2}, \ldots, z_{n-2}\right) d t\right|^{p}+\left|\frac{\partial f(r, z)}{\partial r}\right|^{p}\right) d r d z \\
& \leq c\left(\int_{\mathbb{R}_{+} \times \mathbb{R}^{n-2}} r^{-1}\left|\frac{\partial f}{\partial z_{1}}\right|^{p} d r d z+\left\|\frac{\partial f}{\partial r}\right\|_{L_{p}\left(\mathbb{R}_{+} \times \mathbb{R}^{n-2}\right)}^{p}\right) .
\end{aligned}
$$

Hence, $\left.f\right|_{M}=0$, i.e. $f^{\beta-s, p}$. If $f$ has an arbitrary support then we show that $\left.(\varphi f)\right|_{M}=0$ for every cut-off function $\varphi$ with compact support.

THEOREM 5. Let $u, v_{i j}(i+j \leq\langle s\rangle)$ be arbitrary functions from $W_{p, \beta}^{s}(\mathcal{D})$ and $W_{p, \beta+1 / p}^{s-i-j}\left(\mathbb{R}_{+} \times \mathbb{R}^{n-2}\right)$, respectively. Then

$$
u-\sum_{i+j=0}^{\langle s\rangle} \frac{1}{i ! j !}\left(\mathcal{K} v_{i j}\right)(r, z) y_{1}^{i} y_{2}^{j} \in V_{p, \beta}^{s}(\mathcal{D})
$$

if and only if $v_{i j} \stackrel{\beta-s+i+j, p}{\sim} \stackrel{\circ}{u}_{i j}$ for $i+j \leq\langle s\rangle$.

Proof. 1) Let $v_{i j} \stackrel{\beta-s+i+j, p}{\sim} \stackrel{\circ}{i j}_{i j}$. Then $\mathcal{K}\left(v_{i j}-\stackrel{\circ}{i j}_{i j}\right) \in V_{p, \beta-s+\langle s\rangle+i+j+1+1 / p}^{\langle s\rangle+1}\left(\mathbb{R}_{+}\right.$ $\left.\times \mathbb{R}^{n-2}\right)$ for $i+j \leq\langle s\rangle$. Interpreting $\mathcal{K}\left(v_{i j}-\stackrel{\circ}{u}_{i j}\right)$ as functions on $\mathcal{D}$ we get $\mathcal{K}\left(v_{i j}-\stackrel{\circ}{u}_{i j}\right) \in V_{p, \beta-s+\langle s\rangle+i+j+1}^{\langle s\rangle+1}(\mathcal{D})$ and $\mathcal{K}\left(v_{i j}-\stackrel{\circ}{u}_{i j}\right) y_{1}^{i} y_{2}^{j} \in V_{p, \beta-s+\langle s\rangle+1}^{\langle s\rangle+1}(\mathcal{D}) \subset$ $V_{p, \beta}^{s}(\mathcal{D})$. Using Corollary 2 we obtain (1.19).

2) Assume that (1.19) is satisfied. Then by Corollary 2

$$
\int_{\mathcal{D}} r^{p(\beta-s+\mu+\nu)}\left|D_{y_{1}}^{\mu} D_{y_{2}}^{\nu} \sum_{i+j=0}^{\langle s\rangle}\left(\mathcal{K}\left(\stackrel{\circ}{u}_{i j}-v_{i j}\right)\right) \frac{y_{1}^{i} y_{2}^{j}}{i ! j !}\right|^{p} d x<\infty .
$$

Since $D_{y}^{\gamma} \mathcal{K}\left(\stackrel{\circ}{u}_{i j}-v_{i j}\right) \in V_{p, \beta-s+i+j+|\gamma|}^{0}(\mathcal{D})$ for $|\gamma| \geq 1$ (see Lemma 4), (1.20) yields

$$
\int_{\mathcal{D}} r^{p(\beta-s+\mu+\nu)}\left|\sum_{i+j=0}^{\langle s\rangle-\mu-\nu}\left(\mathcal{K}\left(\stackrel{\circ}{u}_{i+\mu, j+\nu}-v_{i+\mu, j+\nu}\right)\right) \frac{y_{1}^{i} y_{2}^{j}}{i ! j !}\right|^{p} d x<\infty .
$$

For $\mu+\nu=\langle s\rangle,(1.21)$ implies $\mathcal{K}\left(\stackrel{\circ}{u}_{\mu \nu}-v_{\mu \nu}\right) \in V_{p, \beta-s+\mu+\nu}^{0}(\mathcal{D})$, i.e. $\stackrel{\circ}{\mu \nu}_{\mu-s+\mu+\nu, p} \stackrel{\beta}{\sim}$ $v_{\mu \nu}$. Then from (1.21) it follows that

$$
\left.\left.\int_{\mathcal{D}} r^{p(\beta-s+\mu+\nu)}\right|^{\langle s\rangle-1-\mu-\nu} \sum_{i+j=0}\left(\mathcal{K}\left(\stackrel{\circ}{i+\mu, j+\nu}-v_{i+\mu, j+\nu}\right)\right) \frac{y_{1}^{i} y_{2}^{j}}{i ! j !}\right|^{p} d x<\infty .
$$

For $\mu+\nu=\langle s\rangle-1$ this yields $\stackrel{\circ}{\mu \nu}_{\mu-s+\mu+\nu, p}^{\sim} v_{\mu \nu}$. Analogously, by induction on $\mu+\nu$ we show that $\stackrel{\circ}{u \nu}_{\mu-s+\mu+\nu, p}^{\sim} v_{\nu \mu}$ for $\mu+\nu \leq\langle s\rangle-2$. 
1.5. Connection between the spaces of traces. We denote by $B_{p, \beta}^{s-1 / p}\left(\Gamma^{ \pm}\right)(s>$ $1 / p, \beta>-2 / p)$ the space of the traces of functions from $W_{p, \beta}^{s}(\mathcal{D})$ on the sides $\Gamma^{+}$and $\Gamma^{-}$, respectively, provided with the norm

$$
\|u\|_{B_{p, \beta}^{s-1 / p}\left(\Gamma^{ \pm}\right)}=\inf \left\{\|v\|_{W_{p, \beta}^{s}(\mathcal{D})}: v \in W_{p, \beta}^{s}(\mathcal{D}),\left.v\right|_{\Gamma^{ \pm}}=u\right\} .
$$

By Corollary $1, B_{p, \beta}^{s-1 / p}\left(\Gamma^{ \pm}\right) \subset \stackrel{\circ}{s-1 / p}_{p, \beta}^{ \pm}\left(\Gamma^{ \pm}\right)$for $\beta>s-2 / p$. Let $u \in B_{p, \beta}^{s-1 / p}\left(\Gamma^{+}\right)$ and let $v \in W_{p, \beta}^{s}(\mathcal{D})$ be an extension of $u$. Then by Theorem $5, u$ has the representation

$$
u=\left.v\right|_{\Gamma^{+}}=\left.\left(\sum_{i+j \leq\langle s\rangle}\left(\mathcal{K} v_{i j}\right) \frac{y_{1}^{i} y_{2}^{j}}{i ! j !}+v^{\prime}\right)\right|_{\Gamma^{+}}=\sum_{k=0}^{\langle s\rangle}\left(\mathcal{K} v_{k}\right) \frac{r^{k}}{k !}+u^{\prime}
$$

where

$$
v_{k}=\sum_{i=0}^{k}\left(\begin{array}{c}
k \\
i
\end{array}\right)\left(\cos \frac{\omega_{0}}{2}\right)^{i}\left(\sin \frac{\omega_{0}}{2}\right)^{k-i} v_{i, k-i} \in W_{p, \beta+1 / p}^{s-k}\left(\mathbb{R}_{+} \times \mathbb{R}^{n-2}\right)
$$

and $u^{\prime} \in \stackrel{\circ}{B_{p, \beta}^{s-1 / p}}\left(\Gamma^{+}\right)$.

LEMMA 8. Let $v_{k} \in W_{p, \beta+1 / p}^{s-k}\left(\mathbb{R}_{+} \times \mathbb{R}^{n-2}\right)(k=0,1, \ldots,\langle s\rangle)$. Then

$$
\sum_{k=0}^{\langle s\rangle}\left(\mathcal{K} v_{k}\right) \frac{r^{k}}{k !} \in V_{p, \beta-s+1 / p}^{0}\left(\mathbb{R}_{+} \times \mathbb{R}^{n-2}\right) \quad \text { iff } \quad v_{k} \stackrel{\beta-s+k, p}{\sim} 0 .
$$

Proof. 1) If $v_{k} \stackrel{\beta-s+k, p}{\sim} 0$ then $\mathcal{K} v_{k} \in V_{p, \beta-s+k+1 / p}^{0}\left(\mathbb{R}_{+} \times \mathbb{R}^{n-2}\right)$ and $\left(\mathcal{K} v_{k}\right) r^{k} \in$ $V_{p, \beta-s+1 / p}^{0}\left(\mathbb{R}_{+} \times \mathbb{R}^{n-2}\right)$.

2) If $\sum_{k=0}^{\langle s\rangle}\left(\mathcal{K} v_{k}\right) r^{k} / k ! \in V_{p, \beta-s+1 / p}^{0}\left(\mathbb{R}_{+} \times \mathbb{R}^{n-2}\right)$ then from the properties of $\mathcal{K}$ (see Lemma 4) it follows that $\sum_{k=0}^{\langle s\rangle}\left(\mathcal{K} v_{k}\right) r^{k} / k ! \in V_{p, \beta-s+1 / p}^{s}\left(\mathbb{R}_{+} \times \mathbb{R}^{n-2}\right)$, i.e.

$$
\int_{\mathbb{R}_{+} \times \mathbb{R}^{n-2}} r^{p(\beta-s+\mu)+1}\left|D_{r}^{\mu} \sum_{k=0}^{\langle s\rangle}\left(\mathcal{K} v_{k}\right) \frac{r^{k}}{k !}\right|^{p} d r d z<\infty
$$

for $\mu=0,1, \ldots,\langle s\rangle$. Analogously to the proof of Theorem 5 , this implies that $v_{k} \stackrel{\beta-s+k, p}{\sim} 0$.

As a consequence of Lemma 8 we obtain the following theorem.

TheOREM 6. Let $u \in B_{p, \beta}^{s-1 / p}\left(\Gamma^{+}\right)$. Then there exist $v_{k} \in W_{p, \beta+1 / p}^{s-k}\left(\mathbb{R}_{+} \times \mathbb{R}^{n-2}\right)$ $(k=0,1, \ldots,\langle s\rangle)$ such that

$$
u-\sum_{k=0}^{\langle s\rangle}\left(\mathcal{K} v_{k}\right)(r, z) \frac{r^{k}}{k !} \in \stackrel{o}{B}_{p, \beta}^{s-1 / p}\left(\Gamma^{+}\right) .
$$


The functions $v_{k}$ in (1.22) are uniquely determined in the following sense: if $w_{k} \in W_{p, \beta+1 / p}^{s-k}\left(\mathbb{R}_{+} \times \mathbb{R}^{n-2}\right)(k=0,1, \ldots,\langle s\rangle)$ then

$$
u-\sum_{k=0}^{\langle s\rangle}\left(\mathcal{K} w_{k}\right)(r, z) \frac{r^{k}}{k !} \in \stackrel{\circ}{s-1 / p}\left(\Gamma_{p, \beta}^{+}\right) \quad \text { iff } \quad w_{k} \stackrel{\beta-s+k, p}{\sim} v_{k} .
$$

In particular, $v_{k} \stackrel{\beta-s+k, p}{\sim} \partial_{r}^{k} u$ for $k \leq\langle s-1 / p\rangle$ and $v_{k} \stackrel{\beta-s+k, p}{\sim} 0$ for $k>[s-\beta-2 / p]$ (here $[\varkappa]$ denotes the largest integer less than or equal to $\varkappa$, i.e. $[\varkappa]=-\langle-\varkappa\rangle-1$ ).

P r o of. It remains to show that $v_{k} \stackrel{\beta-s+k, p}{\sim} \partial_{r}^{k} u$ for $k \leq\langle s-1 / p\rangle$. Differentiating (1.22) we get

$$
\partial_{r}^{\nu}\left(u-\sum_{k=0}^{\langle s\rangle}\left(\mathcal{K} v_{k}\right) \frac{r^{k}}{k !}\right) \in \stackrel{\circ}{B}_{p, \beta}^{s-\nu-1 / p}\left(\Gamma^{+}\right) .
$$

Since $\partial_{r}^{j} \mathcal{K} v_{k} \in V_{p, \beta+1 / p}^{s-k-j}\left(\Gamma^{+}\right)$for $j \geq 1$ (see Lemma 4) this implies

$$
\partial_{r}^{\nu} u-\sum_{k=0}^{\langle s\rangle-\nu}\left(\mathcal{K} v_{k+\nu}\right) \frac{r^{k}}{k !} \in \stackrel{\circ}{s-\nu-1 / p}_{p, \beta}^{+}\left(\Gamma^{+}\right) .
$$

It can be easily verified that $\mathcal{K}\left(r^{k} v\right) \in V_{p, \beta-k+1 / p}^{s}\left(\mathbb{R}_{+} \times \mathbb{R}^{n-2}\right)$ for $v \in W_{p, \beta+1 / p}^{s}\left(\mathbb{R}_{+}\right.$ $\left.\times \mathbb{R}^{n-2}\right)$ and positive integers $k$. Then (1.23) yields that $\mathcal{K}\left(\partial_{r}^{\nu} u\right)-\mathcal{K} \mathcal{K} v_{\nu} \in$ $V_{p, \beta+\nu+1 / p}^{s}\left(\mathbb{R}_{+} \times \mathbb{R}^{n-2}\right)$, i.e. $\partial_{r}^{\nu} u \stackrel{\beta-s+\nu, p}{\sim} \mathcal{K} v_{\nu} \stackrel{\beta-s+\nu, p}{\sim} v_{\nu}$

The following lemma gives a connection between the spaces $B_{p, \beta}^{s-1 / p}\left(\mathbb{R}_{+} \times\right.$ $\left.\mathbb{R}^{n-2}\right)$ and $W_{p, \beta}^{s-1 / p}\left(\mathbb{R}_{+} \times \mathbb{R}^{n-2}\right)$.

Lemma 9. Let $s-1 / p$ be non-integer, $s>1 / p$ and $\beta>-1 / p$. Then

$$
B_{p, \beta}^{s-1 / p}\left(\mathbb{R}_{+} \times \mathbb{R}^{n-2}\right)=W_{p, \beta}^{s-1 / p}\left(\mathbb{R}_{+} \times \mathbb{R}^{n-2}\right) .
$$

Proof. 1) If $u \in B_{p, \beta}^{s-1 / p}\left(\mathbb{R}_{+} \times \mathbb{R}^{n-2}\right)$ then there exist $v_{k} \in W_{p, \beta+1 / p}^{s-k}\left(\mathbb{R}_{+} \times\right.$ $\mathbb{R}^{n-2}$ ) such that

$$
u-\sum_{k=0}^{\langle s\rangle}\left(\mathcal{K} v_{k}\right) \frac{r^{k}}{k !} \in \stackrel{\circ}{s-1 / p}\left(\mathbb{R}_{p, \beta} \times \mathbb{R}^{n-2}\right)=V_{p, \beta}^{s-1 / p}\left(\mathbb{R}_{+} \times \mathbb{R}^{n-2}\right) .
$$

Since $\left(\mathcal{K} v_{k}\right) r^{k} \in W_{p, \beta}^{s-1 / p}\left(\mathbb{R}_{+} \times \mathbb{R}^{n-2}\right)$ we get $u \in W_{p, \beta}^{s-1 / p}\left(\mathbb{R}_{+} \times \mathbb{R}^{n-2}\right)$.

2) Let $u \in W_{p, \beta}^{s-1 / p}\left(\mathbb{R}_{+} \times \mathbb{R}^{n-2}\right)$. Then analogously to Theorem 5 it can be shown that

$$
v=u-\sum_{k=0}^{\langle s-1 / p\rangle}\left(\mathcal{K} u_{k}\right) \frac{r^{k}}{k !} \in V_{p, \beta}^{s-1 / p}\left(\mathbb{R}_{+} \times \mathbb{R}^{n-2}\right)=\stackrel{o s}{s-1 / p}\left(\mathbb{R}_{p, \beta} \times \mathbb{R}^{n-2}\right)
$$


where $u_{k}=\partial_{r}^{k} u \in W_{p, \beta}^{s-k-1 / p}\left(\mathbb{R}_{+} \times \mathbb{R}^{n-2}\right)$. Without loss of generality we can assume that $\Gamma^{+}$is the half-plane $\Gamma^{+}=\left\{x=(y, z): y_{1}>0, y_{2}=0, z \in \mathbb{R}^{n-2}\right\}$ which can be identified with $\mathbb{R}_{+} \times \mathbb{R}^{n-2}$. If $v^{\prime} \in V_{p, \beta}^{s}(\mathcal{D})$ is an extension of $v$ then

$$
u^{\prime}=v^{\prime}+\sum_{k=0}^{\langle s-1 / p\rangle}\left(\mathcal{K} u_{k}\right) \frac{y_{1}^{k}}{k !}
$$

is an extension of $u$ which lies in $W_{p, \beta}^{s}(\mathcal{D})$. Hence, $u \in B_{p, \beta}^{s-1 / p}\left(\mathbb{R}_{+} \times \mathbb{R}^{n-2}\right)$.

\section{Applications to boundary value problems}

2.1. Compatibility conditions for boundary data on $\Gamma^{ \pm}$. For the spaces $V_{p, \beta}^{s}(\mathcal{D})$ the following lemma has been proved (see [4], [9]).

LEMmA 10. Let $B_{k}^{ \pm}\left(k=1, \ldots, p^{ \pm}\right)$be homogeneous differential operators with constant coefficients of order $m_{k}^{ \pm}<s-1 / p$. Assume that $\left\{B_{k}^{+}\right\}$and $\left\{B_{k}^{-}\right\}$are normal on $\Gamma^{+}$and $\Gamma^{-}$, respectively. Then for all $g_{k}^{ \pm} \in B_{p, \beta}^{s-m_{k}^{ \pm}-1 / p}\left(\Gamma^{ \pm}\right)$there exists $u \in V_{p, \beta}^{s}(\mathcal{D})$ such that $B_{k}^{ \pm} u=g_{k}^{ \pm}$on $\Gamma^{ \pm}$for $k=1, \ldots, p^{ \pm}$.

We will investigate conditions on $g_{k}^{ \pm}\left(g_{k}^{ \pm} \in B_{p, \beta}^{s-m_{k}^{ \pm}-1 / p}\left(\Gamma^{ \pm}\right)\right)$under which there exists $u \in W_{p, \beta}^{s}(\mathcal{D})$ satisfying the boundary conditions

$$
B_{k}^{ \pm} u=\sum_{\mu+\nu+|\gamma|=m_{k}^{ \pm}} b_{k ; \mu \nu \gamma}^{ \pm} D_{y_{1}}^{\mu} D_{y_{2}}^{\nu} D_{z}^{\gamma} u=g_{k}^{ \pm} \quad \text { on } \Gamma^{ \pm}
$$

$\left(k=1, \ldots, p^{ \pm}\right)$. Here we restrict ourselves to the case $n=3$.

By Theorem $6,\left.B_{k}^{ \pm} u\right|_{\Gamma^{ \pm}}-g_{k}^{ \pm}\left(u \in W_{p, \beta}^{s}(\mathcal{D}), g_{k}^{ \pm} \in B_{p, \beta}^{s-m_{k}^{ \pm}-1 / p}\left(\Gamma^{ \pm}\right)\right)$belongs to $B_{p, \beta}^{s-m_{k}^{ \pm}-1 / p}\left(\Gamma^{ \pm}\right)$iff

$$
\partial_{r}^{j}\left(\left.B_{k}^{ \pm} u\right|_{\Gamma^{ \pm}}-g_{k}^{ \pm}\right) \stackrel{\beta-s+m_{k}^{ \pm}+j, p}{\sim} 0
$$

for $j=0,1, \ldots,[s-\beta-2 / p]-m_{k}^{ \pm}$. Using the representation

$$
u=\sum_{i+j=0}^{[s-\beta-2 / p]}\left(\mathcal{K} \stackrel{\circ}{u}_{i j}\right) \frac{y^{i} y^{j}}{i ! j !}+u^{\prime}
$$

where $\stackrel{\circ}{u j}_{i j} \in W_{p, \beta+1 / p}^{s-i-j}\left(\mathbb{R}_{+} \times \mathbb{R}^{n-2}\right), u^{\prime} \in V_{p, \beta}^{s}(\mathcal{D}), D_{r}^{\mu} D_{z}^{\nu} \mathcal{K} \stackrel{\circ}{i j}_{i j} \in V_{p, \beta+1 / p}^{s-i-j-\mu-\nu}\left(\mathbb{R}_{+} \times\right.$ $\mathbb{R}^{n-2}$ ) if $\mu \geq 1$ or $\nu \geq s-\beta-2 / p-i-j$ (see Theorem 5) we get the following equivalences:

$$
\begin{aligned}
\sum_{\mu+\nu+\gamma=m_{k}^{ \pm}}(-i)^{\mu+\nu} b_{k ; \mu \nu \gamma}^{ \pm} \sum_{\sigma=0}^{j}\left(\begin{array}{l}
j \\
\sigma
\end{array}\right)( & \left.\cos \frac{\omega_{0}}{2}\right)^{\sigma}\left( \pm \sin \frac{\omega_{0}}{2}\right)^{j-\sigma} \\
& \times D_{z}^{\gamma} \stackrel{\circ}{u}_{\mu+\sigma, \nu+j-\sigma} \stackrel{\beta-s+m_{k}^{ \pm}+j, p}{\sim^{k}} \partial_{r}^{j} g_{k}^{ \pm}
\end{aligned}
$$


$\left(j=0,1, \ldots,[s-\beta-2 / p]-m_{k}^{ \pm}\right)$. By Lemma 7 the system $(2.3)$ is equivalent to

$$
\begin{aligned}
& \sum_{\mu+\nu+\gamma=m_{k}^{ \pm}}(-i)^{\mu+\nu} b_{k ; \mu \nu \gamma}^{ \pm} \sum_{\sigma=0}^{j}\left(\begin{array}{l}
j \\
\sigma
\end{array}\right)\left(\cos \frac{\omega_{0}}{2}\right)^{\sigma}\left( \pm \sin \frac{\omega_{0}}{2}\right)^{j-\sigma} \\
& \times D_{z}^{\gamma+[s-\beta-2 / p]-m_{k}^{ \pm}-j} \stackrel{\circ}{\mu+\sigma, \nu+j-\sigma}^{\beta-s+[s-\beta-2 / p], p} D_{z}^{[s-\beta-2 / p]-m_{k}^{ \pm}-j} \partial_{r}^{j} g_{k}^{ \pm}
\end{aligned}
$$

$\left(j=0,1, \ldots,[s-\beta-2 / p]-m_{k}^{ \pm}\right) . \quad(2.4)$ can be written as a linear system of equations of the form

$$
\sum_{\mu+\nu=j}^{[s-\beta-2 / p]} c_{\mu, \nu, j, k}^{ \pm} v_{\mu \nu}^{\beta-s+[s-\beta-2 / p], p} D_{z}^{[s-\beta-2 / p]-m_{k}^{ \pm}-j} \partial_{r}^{j} g_{k}^{ \pm}
$$

$\left(j=0,1, \ldots,[s-\beta-2 / p]-m_{k}^{ \pm} ; k=1, \ldots, p^{ \pm}\right)$for the functions $v_{\mu \nu}=$ $D_{z}^{[s-\beta-2 / p]-\mu-\nu} \stackrel{\circ}{u \nu}_{\mu}$. In general, the equations of (2.5) are not linearly independent. Therefore, $(2.5)$ is solvable iff the right-hand sides $D_{z}^{[s-\beta-2 / p]-m_{k}^{ \pm}-j} \partial_{r}^{j} g_{k}^{ \pm}$ of (2.5) satisfy some linear compatibility conditions

$$
\sum_{ \pm} \sum_{k=1}^{p^{ \pm}} \sum_{j=0}^{[s-\beta-2 / p]-m_{k}^{ \pm}} d_{k j \tau}^{ \pm} D_{z}^{[s-\beta-2 / p]-m_{k}^{ \pm}-j} \partial_{r}^{j} g_{k}^{ \pm \beta-s+[s-\beta-2 / p], p} 0
$$

$(\tau=1, \ldots, t)$. The number $t$ of the compatibility conditions depends on the rank of the coefficient matrix of (2.5). If $s-\beta-2 / p$ is not an integer then (2.6) is equivalent to the equations

$$
\left.\sum_{ \pm} \sum_{k=1}^{p^{ \pm}} \sum_{j=0}^{[s-\beta-2 / p]-m_{k}^{ \pm}} d_{k j \tau}^{ \pm} D_{z}^{[s-\beta-2 / p]-m_{k}^{ \pm}-j} \partial_{r}^{j} g_{k}^{ \pm}\right|_{M}=0
$$

$(\tau=1, \ldots, t)$.

THEOREM 7. Suppose that $g_{k}^{ \pm} \in B_{p, \beta}^{s-m_{k}^{ \pm}-1 / p}\left(\Gamma^{ \pm}\right)$satisfy the compatibility conditions (2.6). Then there exists $u \in W_{p, \beta}^{s}(\mathcal{D})$ such that $B_{k}^{ \pm} u=g_{k}^{ \pm}$on $\Gamma^{ \pm}$for $k=1, \ldots, p^{ \pm}$. If $s-\beta-2 / p$ is not an integer then

$$
\|u\|_{W_{p, \beta}^{s}(\mathcal{D})} \leq c \sum_{ \pm} \sum_{k=1}^{p^{ \pm}}\left\|g_{k}^{ \pm}\right\|_{B_{p, \beta}^{s-m_{k}^{ \pm}-1 / p}\left(\Gamma^{ \pm}\right)} .
$$

If $s-\beta-2 / p$ is an integer then

$$
\begin{aligned}
& \|u\|_{W_{p, \beta}^{s}(\mathcal{D})} \leq c\left(\sum_{ \pm} \sum_{k=1}^{p^{ \pm}}\left\|g_{k}^{ \pm}\right\|_{B_{p, \beta}^{s-m_{k}^{ \pm}-1 / p}\left(\Gamma^{ \pm}\right)}\right. \\
& \left.+\sum_{\tau=1}^{t}\left(\int_{0}^{1} \int_{\mathbb{R}} r^{-1}\left|\sum_{ \pm} \sum_{k=1}^{p^{ \pm}} \sum_{j=0}^{[s-\beta-2 / p]-m_{k}^{ \pm}} d_{k j \tau}^{ \pm} D_{z}^{[s-\beta-2 / p]-m_{k}^{ \pm}-j} \partial_{r}^{j} g_{k}^{ \pm}\right| d z d r\right)^{1 / p}\right) .
\end{aligned}
$$


Proof. Under the conditions (2.6) the system (2.5) has a solution $V=$ $\left(v_{\mu \nu}\right)_{\mu+\nu \leq[s-\beta-2 / p]}$ where the functions $v_{\mu \nu}=D_{z}^{[s-\beta-2 / p]-\mu-\nu} \stackrel{\circ}{u}_{\mu \nu}$ are linear combinations of the right-hand sides $D_{z}^{[s-\beta-2 / p]-m_{k}^{ \pm}-j} \partial_{r}^{j} g_{k}^{ \pm}$. Furthermore, the $v_{\mu \nu}$ satisfy

$$
\begin{aligned}
\sum_{\mu+\nu=j}^{[s-\beta-2 / p]} c_{\mu, \nu, j, k}^{ \pm} v_{\mu \nu}= & D_{z}^{[s-\beta-2 / p]-m_{k}^{ \pm}-j} \partial_{r}^{j} g_{k}^{ \pm} \\
& +\sum_{\tau=1}^{t} \alpha_{\tau} \sum_{ \pm} \sum_{j^{\prime}, k^{\prime}} d_{k^{\prime} j^{\prime} \tau}^{ \pm} D_{z}^{[s-\beta-2 / p]-m_{k^{\prime}}^{ \pm}-j^{\prime}} \partial_{r}^{j^{\prime}} g_{k^{\prime}}^{ \pm}
\end{aligned}
$$

with some complex numbers $\alpha_{\tau}$. If we set

$$
w=\sum_{i+j=0}^{[s-\beta-2 / p]}\left(\mathcal{K} \stackrel{\circ}{i j}_{i j}\right) \frac{y_{1}^{i} y_{2}^{j}}{i ! j !}
$$

then $B_{k}^{ \pm} w-g_{k}^{ \pm} \in B_{p, \beta}^{s-m_{k}^{ \pm}-1 / p}\left(\Gamma^{ \pm}\right)$and

$$
\begin{gathered}
\left\|B_{k}^{ \pm} w-g_{k}^{ \pm}\right\|_{\substack{B_{p, \beta}^{s-m_{k}-1 / p}\left(\Gamma^{ \pm}\right) \\
\text {8) }}} \leq c\left(\left\|g_{k}\right\|_{B_{p, \beta}^{s-m_{k}^{ \pm}-1 / p}\left(\Gamma^{ \pm}\right)}^{p}\right. \\
\left.+\sum_{\tau=1}^{t} \int_{0}^{1} \int_{\mathbb{R}} r^{p(\beta-s+[s-\beta-2 / p])+1}\left|\sum_{ \pm} \sum_{j, k^{\prime}} d_{k^{\prime} j \tau}^{ \pm} D_{z}^{[s-\beta-2 / p]-m_{k^{\prime}}^{ \pm j}} \partial_{r}^{j} g_{k^{\prime}}^{ \pm}\right|^{p} d z d r\right) .
\end{gathered}
$$

If $s-\beta-2 / p$ is not an integer then there is a constant $c$ such that the integral in $(2.8)$ is at most $c \sum\left\|g_{k}^{ \pm}\right\|_{B_{p, \beta}^{s-m_{k}^{ \pm}-1 / p}\left(\Gamma^{ \pm}\right)}$. By Lemma 9 there exists $w^{\prime} \in V_{p, \beta}^{s}(\mathcal{D})$ satisfying the equations $B_{k}^{ \pm} w^{\prime}=g_{k}^{ \pm}-B_{k}^{ \pm} w$ on $\Gamma^{ \pm}\left(k=1, \ldots, p^{ \pm}\right)$and the estimate

$$
\left\|w^{\prime}\right\|_{V_{p, \beta}^{s}(\mathcal{D})} \leq c \sum_{ \pm} \sum_{k}\left\|B_{k}^{ \pm} w-g_{k}^{ \pm}\right\|_{B_{p, \beta}^{s-m_{k}^{ \pm}-1 / p}\left(\Gamma^{ \pm}\right)} .
$$

Then $u=w+w^{\prime}$ satisfies the conditions of the theorem.

ExAmple. Let $B^{+} u=u, B^{-} u=u$ and let $g^{+} \in W_{p}^{1-1 / p}\left(\Gamma^{+}\right), g^{-} \in$ $W_{p}^{1-1 / p}\left(\Gamma^{-}\right)$be given functions on $\Gamma^{+}$and $\Gamma^{-}$, respectively.

(a) In the case $p<2$ for arbitrary $g^{+}, g^{-}$there exists $u \in W_{p}^{1}(\mathcal{D})$ such that $\left.u\right|_{\Gamma^{ \pm}}=g^{ \pm}$, and

$$
\|u\|_{W_{p}^{1}(\mathcal{D})} \leq c\left(\left\|g^{+}\right\|_{W_{p}^{1-1 / p}\left(\Gamma^{+}\right)}+\left\|g^{-}\right\|_{W_{p}^{1-1 / p}\left(\Gamma^{-}\right)}\right) .
$$

In this case the space $W_{p}^{1}(\mathcal{D})=W_{p, 0}^{1}(\mathcal{D})$ is imbedded in $V_{p, 0}^{1}(\mathcal{D})$.

(b) In the case $p=2$ the compatibility condition

$$
\int_{0}^{1} \int_{\mathbb{R}} r^{-1}\left|g^{+}(r, z)-g^{-}(r, z)\right|^{2} d z d r<\infty
$$


is necessary and sufficient for the existence of $u \in W_{2}^{1}(\mathcal{D})$ satisfying $\left.u\right|_{\Gamma^{ \pm}}=g^{ \pm}$. This $u$ can be chosen such that

$\|u\|_{W_{2}^{1}(\mathcal{D})}^{2} \leq c\left(\left\|g^{+}\right\|_{W_{2}^{1 / 2}\left(\Gamma^{+}\right)}^{2}+\left\|g^{-}\right\|_{W_{2}^{1 / 2}\left(\Gamma^{-}\right)}^{2}+\int_{0}^{1} \int_{\mathbb{R}}\left|g^{+}(r, z)-g^{-}(r, z)\right|^{2} r^{-1} d z d r\right)$

( $c$ independent of $g^{+}$and $g^{-}$).

(c) If $p>2$ then the condition $\left.g^{+}\right|_{M}=\left.g^{-}\right|_{M}$ is necessary and sufficient for the existence of $u$ satisfying $\left.u\right|_{\Gamma^{ \pm}}=g^{ \pm}$and the inequality (2.9).

For inhomogeneous operators $B_{k}^{ \pm}$with variable coefficients it is more difficult to give a general description of the compatibility conditions on the edge $M$. Therefore, we will restrict ourselves to the case that the coefficients of $B_{k}^{ \pm}$do not depend on the variable $z$, i.e.

$$
B_{k}^{ \pm}=\sum_{\mu+\nu+\gamma \leq m_{k}^{ \pm}} b_{k ; \mu \nu \gamma}^{ \pm}\left(y_{1}, y_{2}\right) D_{y_{1}}^{\mu} D_{y_{2}}^{\nu} D_{z}^{\gamma}
$$

Let $u$ and $g_{k}^{ \pm}$be arbitrary functions from $W_{p, \beta}^{s}(\mathcal{D})$ and $B_{p, \beta}^{s-m_{k}^{ \pm}-1 / p}\left(\Gamma^{ \pm}\right)$, respectively. Then analogously to $(2.3)$ it can be verified that $\left.B_{k}^{ \pm} u\right|_{\Gamma^{ \pm}}-g_{k}^{ \pm} \in$ $\stackrel{\circ}{B}_{p, \beta}^{s-m_{k}^{ \pm}-1 / p}\left(\Gamma^{ \pm}\right)$iff

$$
\begin{gathered}
\left.\sum_{\mu+\nu+\gamma \leq m_{k}^{ \pm}}(-i)^{\mu+\nu} \sum_{\tau=0}^{j}\left(\begin{array}{l}
j \\
\tau
\end{array}\right)\left(\partial_{r}^{j-\tau} b_{k ; \mu \nu \gamma}^{ \pm}\left(r \cos \frac{\omega_{0}}{2}, \pm r \sin \frac{\omega_{0}}{2}\right)\right)\right|_{r=0} \\
\times \sum_{\sigma=0}^{\tau}\left(\begin{array}{l}
\tau \\
\sigma
\end{array}\right)\left(\cos \frac{\omega_{0}}{2}\right)^{\sigma}\left( \pm \sin \frac{\omega_{0}}{2}\right)^{\tau-\sigma} D_{z}^{\gamma} u_{\mu+\sigma, \nu+\tau-\sigma}^{(M)}=\left.\partial_{r}^{j} g_{k}^{ \pm}\right|_{M}
\end{gathered}
$$

$\left(j=0,1, \ldots,[s-\beta-2 / p]-m_{k}^{ \pm}\right)$. Here $u_{\mu \nu}^{(M)}$ denotes the trace of $\partial_{y_{1}}^{\mu} \partial_{y_{2}}^{\nu} u$ on $M$. If $s-\beta-2 / p$ is an integer and $j=[s-\beta-2 / p]-m_{k}^{ \pm}$then (2.10) has to be interpreted in the generalized sense, i.e.

$$
\begin{aligned}
& \left.\sum_{\mu+\nu+\gamma \leq m_{k}^{ \pm}}(-i)^{\mu+\nu} \sum_{\tau=0}^{j}\left(\begin{array}{l}
j \\
\tau
\end{array}\right)\left(\partial_{r}^{j-\tau} b_{k ; \mu \nu \gamma}^{ \pm}\left(r \cos \frac{\omega_{0}}{2}, \pm r \sin \frac{\omega_{0}}{2}\right)\right)\right|_{r=0} \\
& \quad \times \sum_{\sigma=0}^{\tau}\left(\begin{array}{l}
\tau \\
\sigma
\end{array}\right)\left(\cos \frac{\omega_{0}}{2}\right)^{\sigma}\left( \pm \sin \frac{\omega_{0}}{2}\right)^{\tau-\sigma} D_{z}^{\gamma} \stackrel{\circ}{\mu+\sigma, \nu+\tau-\sigma}^{-2 / p, p} \partial_{r}^{j} g_{k}^{ \pm} .
\end{aligned}
$$

By means of the equations (2.10) one can prove the following lemma which was used in [10].

LEMMA 11. Let $\chi$ be a smooth function on $\overline{\mathcal{D}}$ with compact support such that $D_{y}^{\alpha} \chi=0$ on $M$ for $|\alpha| \geq 1$ (the last condition is satisfied, e.g., if $\chi(y, z)=$ $\chi_{1}(y) \chi_{2}(z)$ and $\chi_{1}=c$ in a neighbourhood of $\left.y=0\right)$. If $u \in W_{p, \beta}^{s}(\mathcal{D})$ and $B_{k}^{ \pm} u=$ 0 on $\Gamma^{ \pm} \cap \operatorname{supp} \chi$ then there exists $v \in W_{p, \beta}^{s}(\mathcal{D})$ such that $\operatorname{supp} v \subset \operatorname{supp} \chi$, 
$\partial v / \partial z \in W_{p, \beta}^{s}(\mathcal{D}), B_{k}^{ \pm} v=B_{k}^{ \pm}(\chi u)$ on $\Gamma^{ \pm}$and

$$
\|v\|_{W_{p, \beta}^{s}(\mathcal{D})}+\left\|\frac{\partial v}{\partial z}\right\|_{W_{p, \beta}^{s}(\mathcal{D})} \leq c\|u\|_{W_{p, \beta}^{s}(\mathcal{D})} .
$$

In order to prove this we need the following two lemmas.

LEMMA 12. Consider the differential equation

$$
p_{1}\left(D_{z}\right) u_{1}+p_{2}\left(D_{z}\right) u_{2}+\ldots+p_{n}\left(D_{z}\right) u_{n}=0
$$

for the functions $u_{j} \in W_{p}^{s+t_{j}}(\mathbb{R})\left(t_{j}\right.$ an integer, $\left.t_{j} \geq 0\right)$ where $p_{j}\left(D_{z}\right)$ are differential operators with ord $p_{j} \leq t_{j}$ and constant coefficients. Then there exist nonnegative integers $\tau_{1}, \ldots, \tau_{n-1}$ and differential oprators $q_{i j}\left(D_{z}\right)(i=1, \ldots, n ; j=$ $1, \ldots, n-1)$ with ord $q_{i j} \leq \tau_{j}-t_{i}\left(q_{i j}=0\right.$ if $\left.\tau_{j}<t_{i}\right)$ and constant coefficients such that the general solution $U=\left(u_{1}, \ldots, u_{n}\right)^{T} \in \prod W_{p}^{s+t_{j}}(\mathbb{R})$ of $(2.12)$ can be written in the form

$$
\left(\begin{array}{c}
u_{1} \\
\vdots \\
u_{n}
\end{array}\right)=\left(\begin{array}{lll}
q_{1,1} & \cdots & q_{1, n-1} \\
\vdots & & \vdots \\
q_{n, 1} & \cdots & q_{n, n-1}
\end{array}\right)\left(\begin{array}{l}
\varphi_{1} \\
\vdots \\
\varphi_{n-1}
\end{array}\right)
$$

where $\varphi_{j}$ are arbitrary functions from $W_{p}^{s+\tau_{j}}(\mathbb{R})(j=1, \ldots, n-1)$.

Proof. We use induction on $\min$ ord $p_{j}=\mu$.

1) Let first $\mu=0$. Without loss of generality we can assume that $t_{n}-\operatorname{ord} p_{n}=$ $\ldots=t_{l}-\operatorname{ord} p_{l}<t_{l-1}-\operatorname{ord} p_{l-1} \leq \ldots \leq t_{1}-\operatorname{ord} p_{1}$ and $\operatorname{ord} p_{n} \leq \operatorname{ord} p_{n-1} \leq$ $\cdots \leq \operatorname{ord} p_{l}$.

1 (a) If ord $p_{n}=0, p_{n} \neq 0$, then the general solution $U=\left(u_{1}, \ldots, u_{n}\right)^{T} \in$ $\prod W_{p}^{s+t_{j}}(\mathbb{R})$ can be written in the form

$$
\begin{aligned}
& u_{1}=q_{11} \varphi_{1}=\varphi_{1}, \ldots, u_{n-1}=q_{n-1, n-1} \varphi_{n-1}=\varphi_{n-1}, \\
& u_{n}=-\frac{1}{p_{n}}\left(p_{1} \varphi_{1}+\ldots+p_{n-1} \varphi_{n-1}\right) \quad\left(\varphi_{j} \in W_{p}^{s+t_{j}}(\mathbb{R})\right) .
\end{aligned}
$$

1(b) If ord $p_{n}>0$ then there exists $k \in\{1, \ldots, l-1\}$ such that ord $p_{k}=0$. In this case we use induction on $d=t_{k}-\operatorname{ord} p_{k}-\left(t_{n}-\operatorname{ord} p_{n}\right)$.

Assume that the assertion is true if $t_{k}-\operatorname{ord} p_{k}-\left(t_{n}-\operatorname{ord} p_{n}\right)<d$. Using the representation $p_{j}=p_{n} p_{j}^{\prime}+r_{j}(j=l, \ldots, n-1)$ where ord $p_{j}^{\prime}=\operatorname{ord} p_{j}-\operatorname{ord} p_{n}$ and ord $r_{j}<$ ord $p_{n}$ we obtain

$$
\begin{aligned}
p_{1} u_{1}+\ldots+p_{l-1} u_{l-1}+r_{l} u_{l}+ & \ldots+r_{n-1} u_{n-1} \\
& +p_{n}\left(u_{n}+p_{l}^{\prime} u_{l}+\ldots+p_{n-1}^{\prime} u_{n-1}\right)=0 .
\end{aligned}
$$

Since $t_{j}-\operatorname{ord} p_{j} \geq t_{n}-\operatorname{ord} p_{n}+1$ for $j=1, \ldots, l-1$ and $t_{j}-\operatorname{ord} r_{j} \geq t_{n}-\operatorname{ord} p_{n}+1$ for $j=l, \ldots, n-1$ we get

$$
p_{1} u_{1}+\ldots+p_{l-1} u_{l-1}+r_{l} u_{l}+\ldots+r_{n-1} u_{n-1} \in W_{p}^{s+t_{n}+1-\operatorname{ord} p_{n}}(\mathbb{R}) .
$$


Hence, $v_{n}=u_{n}+p_{l}^{\prime} u_{l}+\ldots+p_{n-1}^{\prime} u_{n-1} \in W_{p}^{s+t_{n}^{\prime}}(\mathbb{R})$ where $t_{n}^{\prime}=t_{n}+1$, i.e. $t_{k}-\operatorname{ord} p_{k}-\left(t_{n}^{\prime}-\operatorname{ord} p_{n}\right)=d-1$. Then by the inductive assumption there exist non-negative integers $\tau_{1}, \ldots, \tau_{n-1}$ and differential operators $q_{i j}(i=1, \ldots, n-$ 1), $q_{n j}^{\prime}$ with ord $q_{i j} \leq \tau_{j}-t_{i}$, ord $q_{n j}^{\prime} \leq \tau_{j}-t_{n}^{\prime}$ such that the general solution $\left(u_{1}, \ldots, u_{n-1}, v_{n}\right) \in \prod_{j=1}^{n-1} W_{p}^{s+t_{j}}(\mathbb{R}) \times W_{p}^{s+t_{n}^{\prime}}(\mathbb{R})$ of $(2.14)$ is

$$
u_{i}=\sum_{j=1}^{n-1} q_{i j} \varphi_{j}, \quad v_{n}=\sum_{j=1}^{n-1} q_{n j}^{\prime} \varphi_{j} \quad\left(\varphi_{j} \in W_{p}^{s+\tau_{j}}(\mathbb{R})\right)
$$

Hence,

$$
\begin{aligned}
& u_{i}=\sum_{j=1}^{n-1} q_{i j} \varphi_{j} \\
& u_{n}=v_{n}-p_{l}^{\prime} u_{l}-\ldots-p_{n-1}^{\prime} u_{n-1}=\sum_{j=1}^{n-1}\left(q_{n j}^{\prime}-\sum_{i=l}^{n-1} p_{i}^{\prime} q_{i j}\right) \varphi_{j} .
\end{aligned}
$$

2) Let $\mu=\min _{j}$ ord $p_{j} \geq 1$. Without loss of generality we assume that ord $p_{n} \leq$ $\ldots \leq \operatorname{ord} p_{1}$. Then each $p_{j}$ has a representation $p_{j}=p_{n} p_{j}^{\prime}+r_{j}$ where ord $p_{j}^{\prime}=$ ord $p_{j}-\operatorname{ord} p_{n}, \operatorname{ord} r_{j}<$ ord $p_{j}$. If $r_{j}=0$ for $j=2, \ldots, n$ then (2.12) is equivalent to $u_{1}+p_{2}^{\prime} u_{2}+\ldots+p_{n}^{\prime} u_{n}=0$ and the assertion of the lemma follows from the first part of the proof. If e.g. $r_{n-1} \neq 0$ then we write $(2.12)$ in the form

$$
p_{1} u_{1}+\ldots+p_{n-2} u_{n-2}+r_{n-1} u_{n-1}+p_{n}\left(u_{n}+p_{n-1}^{\prime} u_{n-1}\right)=0 .
$$

Since ord $r_{n-1}<\mu$ we can suppose that there exist integers $\tau_{1}^{\prime}, \ldots, \tau_{n-1}^{\prime}$ and operators $q_{i j}^{\prime}$, ord $q_{i j}^{\prime} \leq \tau_{j}^{\prime}-t_{i}$, such that the general solution of $(2.15)$ is

$$
u_{i}=\sum_{j=1}^{n-1} q_{i j}^{\prime} \psi_{j} \quad(i=1, \ldots, n-1), \quad u_{n}+p_{n-1}^{\prime} u_{n-1}=\sum_{j=1}^{n-1} q_{n j}^{\prime} \psi_{j}
$$

$\left(\psi_{j} \in W_{p}^{s+\tau_{j}^{\prime}}(\mathbb{R})\right)$. From the last equation it follows that

$$
u_{n}+\sum_{j=1}^{n-1}\left(p_{n-1}^{\prime} q_{n-1, j}^{\prime}-q_{n j}^{\prime}\right) \psi_{j}=0 .
$$

Hence, by the first part of the proof there exist integers $\tau_{1}, \ldots, \tau_{n-1}$ and operators $q_{i j}^{\prime \prime}\left(\right.$ ord $q_{i j}^{\prime \prime} \leq \tau_{j}-\tau_{i}^{\prime}$ for $i=1, \ldots, n-1$; ord $\left.q_{n j}^{\prime \prime} \leq \tau_{j}-t_{n}\right)$ such that the general solution of (2.16) can be written in the form

$$
\psi_{i}=\sum_{j=1}^{n-1} q_{i j}^{\prime \prime} \varphi_{j} \quad(i=1, \ldots, n-1), \quad u_{n}=\sum_{j=1}^{n-1} q_{n j}^{\prime \prime} \varphi_{j}
$$

$\left(\varphi_{j} \in W_{p}^{s+\tau_{j}}(\mathbb{R})\right)$. This implies the assertion of the lemma.

By induction on the number of equations Lemma 12 can be easily generalized to systems of differential equations with constant coefficients. 
LEMma 13. Consider a system of ordinary differential equations

$$
\begin{gathered}
p_{11}\left(D_{z}\right) u_{1}+\ldots+p_{1 n}\left(D_{z}\right) u_{n}=0, \\
\vdots \\
p_{m 1}\left(D_{z}\right) u_{1}+\ldots+p_{m n}\left(D_{z}\right) u_{n}=0,
\end{gathered}
$$

where $p_{i j}$ are differential operators with constant coefficients and ord $p_{i j} \leq s_{i}+t_{j}$. Then there exist an integer $l, 0 \leq l \leq n-1$, integers $\tau_{1}, \ldots, \tau_{l}$ and differential operators $q_{i j}(i=1, \ldots, n ; j=1, \ldots, l)$ with ord $q_{i j} \leq \tau_{j}-t_{i}$ and constant coefficients such that the general solution $\left(u_{1}, \ldots, u_{n}\right) \in \prod W_{p}^{s+t_{i}}(\mathbb{R})$ can be written in the form

$$
u_{i}=\sum_{j=1}^{l} q_{i j} \varphi_{j}
$$

where $\varphi_{j}$ are arbitrary functions from $W_{p}^{s+\tau_{j}}(\mathbb{R})$.

P r o of. Suppose that the assertion is true for every system of $m-1$ equations. By Lemma 12 the general solution of the last equation of (2.17) has a representation $u_{i}=\sum_{j=1}^{n-1} q_{i j}^{\prime} \psi_{j}(i=1, \ldots, n-1)$ where $\psi_{j} \in W_{p}^{s+\tau_{j}^{\prime}}(\mathbb{R})$, ord $q_{i j}^{\prime} \leq \tau_{j}^{\prime}-t_{i}$. Replacing $u_{j}$ by $\sum q_{i j}^{\prime} \psi_{j}$ in the first $m-1$ equations of (2.17) we get the system of $m-1$ equations

$$
\sum_{j=1}^{n-1}\left(\sum_{k=1}^{n} p_{i k} q_{k j}^{\prime}\right) \psi_{j}=0 \quad(i=1, \ldots, m-1) .
$$

Applying the inductive assumption to this system we get the assertion of the lemma.

Remark 5. For given $u_{j} \in W_{p}^{s+t_{j}}(\mathbb{R})$ the functions $\varphi_{j}$ in Lemma 13 can be chosen such that

$$
\sum_{j=1}^{l}\left\|\varphi_{j}\right\|_{W_{p}^{s+\tau_{j}}(\mathbb{R})} \leq c \sum_{j=1}^{n}\left\|u_{j}\right\|_{W_{p}^{s+t_{j}}(\mathbb{R})}
$$

where the constant $c$ is independent of $u_{1}, \ldots, u_{n}$.

Proof of Lemma 11. First, suppose $s-\beta-2 / p$ is not an integer. Then the traces $u_{i j}^{(M)}$ of $\partial_{y_{1}}^{i} \partial_{y_{2}}^{j} u$ on $M$ satisfy the differential equations (2.10) where $g_{k}^{ \pm}=0$. We write $(2.10)$ in the form

$$
\mathcal{P} U=0
$$

where $\mathcal{P}$ is a matrix of ordinary differential operators with constant coefficients and $U$ is the vector composed of the functions $u_{i j}^{(M)} \in W_{p}^{s-i-j-\beta-2 / p}(M)(i+j \leq$ $\langle s-\beta-2 / p\rangle)$. By Lemma 13 there exist a vector $\Phi$ of functions $\varphi_{1}, \ldots, \varphi_{l}$ and a matrix $Q$ of differential operators such that $U=Q \Phi$. Another solution of (2.20) 
is $U^{\prime}=Q\left(\left.\chi\right|_{M} \Phi\right)$. If we set

$$
V=\left(v_{i j}\right)=-\left[Q,\left.\chi\right|_{M}\right] \Phi=\left.\chi\right|_{M} Q \Phi-Q\left(\left.\chi\right|_{M} \Phi\right)
$$

$([Q, \chi]$ denotes the commutator of $Q$ and $\chi)$ then we get

$$
\mathcal{P}\left(V-\left.\chi\right|_{M} U\right)=\mathcal{P}\left(\left.\chi\right|_{M} Q \Phi\right)-\mathcal{P} Q\left(\left.\chi\right|_{M} \Phi\right)-\mathcal{P}\left(\left.\chi\right|_{M} U\right)=0 .
$$

Furthermore, from the representation $(2.21)$ and Remark 5 it follows that $v_{i j} \in$ $W_{p}^{s-i-j+1-\beta-2 / p}(M)$ and

$$
\left\|v_{i j}\right\|_{W_{p}^{s-i-j+1-\beta-2 / p}(M)} \leq c \sum_{\mu+\nu=0}^{\langle s-\beta-2 / p\rangle}\left\|u_{\mu \nu}^{(M)}\right\|_{W_{p}^{s-\mu-\nu-\beta-2 / p}(M)} .
$$

We define the functions $\widetilde{v}, g_{k}^{ \pm}$as follows:

$$
\widetilde{v}=\sum_{i+j=0}^{\langle s-\beta-2 / p\rangle}\left(\mathfrak{K} v_{i j}\right) \frac{y_{1}^{i} y_{2}^{j}}{i ! j !}, \quad g_{k}^{ \pm}=B_{k}^{ \pm}(\widetilde{v}-\chi u) .
$$

Since $v_{i j} \in W_{p}^{s-i-j+1-\beta-2 / p}(M)$ and $B_{k}^{ \pm}(\chi u)=\left[B_{k}^{ \pm}, \chi\right] u \in B_{p, \beta}^{s+1-m_{k}^{ \pm}-1 / p}\left(\Gamma^{ \pm}\right)$, $g_{k}^{ \pm}$belongs to $B_{p, \beta}^{s+1-m_{k}^{ \pm}-1 / p}\left(\Gamma^{ \pm}\right)$. Furthermore, (2.22) implies $\left.\partial_{r}^{i} g_{k}^{ \pm}\right|_{M}=0$ and $\left.\partial_{z} \partial_{r}^{j} g_{k}^{ \pm}\right|_{M}=0$ for $j=0,1, \ldots,\langle s-\beta-2 / p\rangle-m_{k}^{ \pm}$. Consequently, $g_{k}^{ \pm}$and $\partial_{z} g_{k}^{ \pm}$ belong to $\stackrel{\circ}{B}_{p, \beta}^{s-m_{k}^{ \pm}-1 / p}\left(\Gamma^{ \pm}\right)$. Then by Lemma 3.1 of [4] there exists $w \in V_{p, \beta}^{s}(\mathcal{D})$ satisfying $B_{k}^{ \pm} w=g_{k}^{ \pm}$on $\Gamma^{ \pm}, B_{k}^{ \pm} \partial w / \partial z=\partial_{z} g_{k}^{ \pm}$on $\Gamma^{ \pm}$and

$$
\begin{aligned}
\|w\|_{V_{p, \beta}^{s}(\mathcal{D})}+\| \frac{\partial w}{\partial z} & \|_{V_{p, \beta}^{s}(\mathcal{D})} \\
& \leq c \sum_{ \pm} \sum_{k=1}^{p}\left(\left\|g_{k}^{ \pm}\right\|_{B_{p, \beta}^{s-m_{k}-1 / p}\left(\Gamma^{ \pm}\right)}+\left\|\partial_{z} g_{k}^{ \pm}\right\|_{B_{p, \beta}^{s-m_{k}-1 / p}\left(\Gamma^{ \pm}\right)}^{ \pm}\right) .
\end{aligned}
$$

Therefore, $v=\widetilde{v}-w$ satisfies the conditions of Lemma 11. Similarly the lemma can be proved for $s-\beta-2 / p$ an integer.

2.2. Regularity of solutions of elliptic boundary value problems. We consider the boundary value problem

$$
\begin{aligned}
L u & =\sum_{|\alpha| \leq 2 m} a_{\alpha}(x) D^{\alpha} u=f \quad \text { in } \mathcal{D}, \\
B_{k}^{ \pm} u & =\sum_{|\alpha| \leq m_{k}^{ \pm}} b_{k ; \alpha}^{ \pm}(x) D^{\alpha} u=g_{k}^{ \pm} \quad \text { on } \Gamma^{ \pm}
\end{aligned}
$$

$(k=1, \ldots, m)$ where $L$ is an elliptic differential operator with smooth coefficients and $\left\{B_{k}^{+}\right\},\left\{B_{k}^{-}\right\}$are normal systems of boundary operators with smooth coefficients which cover the operator $L$ on $\Gamma^{+}$and $\Gamma^{-}$, respectively.

The following regularity assertion has been proved in [4] (see also [9]). 
LEMma 14. Let $u \in V_{p, \beta}^{2 m}(\mathcal{D})$ be a solution of the boundary value problem (2.23) where $f \in V_{p, \beta+s}^{s}(\mathcal{D}), g_{k}^{ \pm} \in \stackrel{\circ}{B}_{p, \beta+s}^{s-2 m-m_{k}^{ \pm}-1 / p}\left(\Gamma^{ \pm}\right)$. Assume that the support of $u$ is compact. Then $u \in V_{p, \beta+s}^{s+2 m}(\mathcal{D})$.

By using Theorems 5 and 6 this assertion can be extended to the spaces $W_{p, \beta}^{s}$.

TheOREM 8. Let $u \in W_{p, \beta}^{2 m}(\mathcal{D})(\beta>-2 / p)$ be a solution of the boundary value problem (2.23) with compact support. If $f \in W_{p, \beta+s}^{s}(\mathcal{D})$ and $g_{k}^{ \pm} \in$ $B_{p, \beta+s}^{s+2 m-m_{k}^{ \pm}-1 / p}\left(\Gamma^{ \pm}\right)$then $u \in W_{p, \beta+s}^{s+2 m}(\mathcal{D})$.

Proof. By Theorem 5 the function $u \in W_{p, \beta}^{2 m}(\mathcal{D})$ is the sum of a function

$$
u_{1}=\sum_{i+j=0}^{2 m-1}\left(\mathcal{K} \stackrel{\circ}{i j}_{j}\right) \frac{y_{1}^{i} y_{2}^{j}}{i ! j !} \in W_{p, \beta+s}^{s+2 m}(\mathcal{D}) \subset W_{p, \beta}^{2 m}(\mathcal{D})
$$

$\left(\stackrel{\circ}{u}_{i j} \in W_{p, \beta+1 / p}^{2 m-i-j}\left(\mathbb{R}_{+} \times \mathbb{R}^{n-2}\right)\right)$ and a function $u_{2} \in V_{p, \beta}^{2 m}(\mathcal{D})$. Obviously,

$$
\begin{aligned}
L u_{2} & =f-L u_{1} \in W_{p, \beta+s}^{s}(\mathcal{D}) \cap V_{p, \beta}^{0}(\mathcal{D})=V_{p, \beta+s}^{s}(\mathcal{D}), \\
B_{k}^{ \pm} u_{2} & =g_{k}^{ \pm}-B_{k}^{ \pm} u_{1} \in B_{p, \beta+s}^{s+2 m-m_{k}^{ \pm}-1 / p}\left(\Gamma^{ \pm}\right) \cap B_{p, \beta}^{2 m-m_{k}^{ \pm}-1 / p}\left(\Gamma^{ \pm}\right) \\
& =B_{p, \beta+s}^{s+2 m-m_{k}^{ \pm}-1 / p}\left(\Gamma^{ \pm}\right) .
\end{aligned}
$$

Therefore, from Lemma 14 it follows that $u_{2} \in V_{p, \beta+s}^{s+2 m}(\mathcal{D})$ and $u=u_{1}+u_{2} \in$ $W_{p, \beta+s}^{s+2 m}(\mathcal{D})$.

\section{References}

[1] P. Grisvard, Elliptic Problems in Nonsmooth Domains, Pitman, Boston 1985.

[2] V. A. Kondrat'ev, Boundary value problems for elliptic equations on domains with conical or corner points, Trudy Moskov. Mat. Obshch. 26 (1967), 209-292 (in Russian).

[3] V. G. Maz'ya and B. A. Plamenevskiǔ, $L_{p}$ and Hölder estimates and the MirandaAgmon maximum principle for solutions of elliptic boundary value problems in a domain with singular points on the boundary, Math. Nachr. 81 (1978), 25-82 (in Russian).

[4] -, - , L L-estimates for elliptic boundary value problems in domains with edges, Trudy Moskov. Mat. Obshch. 37 (1978), 49-93 (in Russian).

[5] - - - Weighted spaces with inhomogeneous norms and boundary value problems in domains with conical points, in: Vorträge der Tagung "Elliptische Differentialgleichungen", Rostock 1977, 161-190 (in Russian).

[6] V. G. Maz'ya und J. Roßmann, Über die Asymptotik der Lösungen elliptischer Randwertaufgaben in der Umgebung von Kanten, Math. Nachr. 138 (1988), 27-53.

[7] V. A. Nikishkin, Singularities of the solution of the Dirichlet problem for second order elliptic differential equations in the neighbourhood of an edge, Vestnik Moskov. Univ. Ser. I Mat. Mekh. 34 (2) (1979), 51-62 (in Russian).

[8] J. Roßman, Über zwei Klassen von gewichteten Sobolevräumen in ebenen Gebieten mit Ecken und Anwendungen auf elliptische Randwertaufgaben, Rostock. Math. Kolloq. 29 (1986), 78-98. 
[9] J. Roßman, Gewichtete Sobolev-Slobodezkij-Räume und Anwendungen auf elliptische Randwertaufgaben in Gebieten mit Kanten, doctoral thesis, University of Rostock, 1988.

[10] - , The asymptotics of the solutions of linear elliptic variational problems near edges, Anal. Anwendungen 9 (6) (1990), 565-578.

[11] H. Triebel, Interpolation Theory, Function Spaces, Differential Operators, Deutscher Verlag Wiss., Berlin 1978. 